\title{
HOMOGENEOUS EXTENSIONS OF POSITIVE LINEAR OPERATORS
}

\author{
BY \\ DOROTHY MAHARAM
}

1. Introduction. A positive linear operator is (roughly speaking) a countably additive, order preserving, $\sigma$-finite linear mapping $\phi$ from one function space, $F$, to another, $F^{\prime} \cdot{ }^{1}$ ) (For precise definitions, see $\$ 2$ below. We assume in particular that $F$ and $F^{\prime}$ satisfy the countable chain condition.) It has been shown in [4] that a normal form representation can be given for $\phi$ : if the function space $F^{\prime}$ consists of the "measurable" functions (modulo "null" functions) on a space $X$, then $F$ is isomorphic to a subspace of the space $F^{*}$ of "measurable" functions on $X \times Y$, where $Y$ is an ordinary numerical measure space, and $\phi$ can be extended to a positive linear operator $\phi^{*}$ from $F^{*}$ to $F^{\prime}$, in such a way that (to within the isomorphism mentioned) $\phi^{*} f=g$, where $g(x)=\int_{Y} f(x, y) d y$.

We are concerned here with the case in which $F^{\prime}=F$. It is now of importance (for instance in ergodic theory) to consider the iterates of $\phi$; and the normal form representation just mentioned has now the drawback that the isomorphism imbedding $F$ in $F^{*}$ interferes with the description of these iterates. The present paper takes a first step towards obtaining a more satisfactory representation of $\phi$ and its iterates.

Given a positive linear operator $\phi$ from $F$ to $F$, we shall show (Theorem 1, 4.1) that the function space $F$ can be imbedded in a larger space $F^{*}$, and the operator $\phi$ extended to a positive linear operator $\phi^{*}$ from $F^{*}$ to $F^{*}$, in such a way that the extended operator has the following property, which we call "full homogeneity": For each characteristic function $\chi \in F^{*}$, and each function $g \in F^{*}$ such that $0 \leqq g \leqq \phi^{*} \chi$, there exists a characteristic function $\chi^{\prime}$ in $F^{*}$ such that $\chi^{\prime} \leqq \chi$ and $\left.\phi^{*} \chi^{\prime}=g .{ }^{2}\right)$ It follows that the iterates of the extended operator $\phi^{*}$ will also be fully homogeneous, and therefore $\sigma$-finite. (Even in simple cases, the iterates of $\phi$ itself may fail to be $\sigma$-finite $\left({ }^{3}\right)$.) A routine application of the results of [4] would then lead easily to a simultaneous representation theorem for $\phi$ and its iterates; however, a sharper theorem can be

Received by the editors October 8, 1959.

(1) Or from one conditionally complete vector $\sigma$-lattice, satisfying the countable chain condition, to another; cf. [4, p. 156].

(2) To improve the legibility of formulae, we often omit brackets, writing (as here) $\phi^{*} \chi$ for $\phi^{*}(x)$, and later $R x$ for $R(x)$, etc.

(3) For example, let $F$ be the space of measurable functions $f(x, y)$ modulo null functions on the plane (with ordinary measure), and let $\phi f=g$ where $g(x, y)=\int_{-\infty}^{\infty} f(x, t) d t$ (independent of $y$ ). Then $\phi$ is an $F$-integral on $F$ (and in particular is $\sigma$-finite), but if $f \in F^{+}$and $\phi^{2} f$ is finite, then $f=0$, whence $\phi^{2}$ is not $\sigma$-finite. 
deduced with more trouble, so we leave this application for a later paper. Meanwhile we show (Theorem 3, §7) that when $F$ arises from a genuine numerical measure (that is, $F$ is the space of measurable functions modulo null functions on a measure space) then the extended space $F^{*}$ in Theorem 1 can also be taken to arise from a numerical measure. The deduction of Theorem 3 depends on a property of measure algebras (Theorem $2, \S 6$ ) which may be of independent interest: Given a $\sigma$-subalgebra $A$ of a measure algebra $(E, \mu)$, and given a $\sigma$-finite measure $\nu$ on $A$ which is equivalent to $\mu$ on $A$ (that is, $\nu$ vanishes only for the zero element of $A$ ), there exists a $\sigma$-finite measure $\nu^{*}$ on $E$ which extends $\nu$ on $A$ and is equivalent to $\mu$ on $E$.

The technique employed for the proofs makes considerable use of representation spaces and of continuous functions on them; thus, after giving the notation and some preliminary results in $\S 2$, we collect some results on the representation spaces of an algebra and a complete subalgebra of it in $\$ 3$. Theorem 1 and its proof occupy $\S \S 4$ and 5 , Theorem 2 is dealt with in $\S 6$, and Theorem 3 in $\$ 7$. The background material and a few specific results are quoted without proof from [2-5]; apart from this the present paper is largely self-contained.

\section{Notation and preliminaries.}

2.1 Algebras and representation spaces. In general we use the same notations as in $[2 ; 4]$, an acquaintance with which is assumed. The term "algebra" always means "Boolean algebra"; if $E$ is an algebra, the symbols $o$ and $e$ denote the zero and unit elements of $E$ respectively, and $-x$ denotes the complement of $x \in E$; and the symmetric difference of $x, y \in E$ (written $x+{ }_{2} y$ in $\left.[2 ; 4]\right)$ is here written as $x+y$.

The representation space of an algebra $E$ is the space $R$ of ultrafilters on $E$; to each $x \in E$ corresponds the set $R x \subset R$ consisting of those ultrafilters which contain $x$ (thus $R o=\phi, R e=R$ ), and $T$ is topologised by taking the sets $R x$ as a basis; $R$ is compact Hausdorff, and each $R x$ is both open and closed. The correspondence $x \leftrightarrow R x$ is a finite isomorphism between $E$ and the algebra of all open-closed subsets of $R$. We write $B^{*} R$ for the family of Borel subsets of $R, B R$ for the family of "restricted Borel sets" (the Borel field generated by the open-closed sets of $R$ ), and $\nVdash R$ for the family of first category subsets of $R$. We have:

(1) If $X \in B^{*} R, X=G+H$ where $G$ is open and $H \in \nVdash R$.

(Here again + denotes symmetric difference.) As the method of proof of (1) ("Borel induction") will be used frequently in what follows, we sketch it: The family of all sets of the form $G+H$, where $G$ is open and $H \in \Re R$, is closed under complementation and under countable unions; hence it is a Borel field containing all open sets, and so it contains $B^{*} R$.

Similarly, if $E$ is a $\sigma$-algebra, we have

(2) If $X \in ß R, X=R x+H$ where $x \in E$ and $H \in \mathscr{K} R$.

It follows that the $\sigma$-algebra $E$ is isomorphic to $B R / \mathcal{K} R$; if further $E$ is a 
complete algebra (as it will be if it satisfies the countable chain condition) then this $\sigma$-isomorphism is necessarily complete. If $E$ is complete, (2) applies to all sets in $B^{*} R$, so that $E$ is (completely) isomorphic to $B^{*} R / \mathscr{K} R$.

2.2 Subalgebras. Let $A$ be a subalgebra of an arbitrary algebra $E$, and let $S, R$ be their respective representation spaces. There is a natural mapping $\xi$ of $R$ onto $S$, obtained as follows: each point of $R$ is an ultrafilter $U$ on $E$, and its trace $\mathcal{U} \cap A$ on $A$ is an ultrafilter on $A$; we take $\xi(\mathcal{U})=\mathcal{U} \cap A$. It is easily verified that

$$
\xi^{-1}(S y)=R y, \quad(y \in A),
$$

so that $\xi$ is continuous. If further $B$ is a subalgebra of $A$, with $T$ as its representation space, and $\xi^{\prime}, \xi^{\prime \prime}$ are the corresponding mappings from $R$ to $T, S$ to $T$, then clearly

$$
\xi^{\prime}=\xi^{\prime \prime} \xi .
$$

Let $B_{o}$ be the Borel field (of subsets of $R$ ) generated by the sets $R y$, $y \in B$; a "Borel induction" argument shows that

$$
B R \supset B_{0}=\xi^{-1} B S \text {. }
$$

2.3 Complete subalgebras. A subalgebra $A$ of an arbitrary algebra $E$ will be called a complete subalgebra of $E$ if, for every $H \subset A$, the supremum $\mathrm{V} H=\mathrm{V}\{h \mid h \in H\}$ of $H$ in $E$ exists and belongs to $A$; thus $A$ (but not necessarily $E$ ) is then itself a complete algebra. An isomorphism $\theta$ of an algebra $B$ onto a subalgebra $A$ of $E$ is "complete with respect to $E$ " if $A$ is a complete subalgebra of $E$; this implies that $B$ is itself a complete algebra and that, for every $H \subset B, \theta(\mathrm{V} H)=\mathrm{V}(\theta H)$ (the supremum in $A$ or $E$ ).

Now suppose $A$ is a complete subalgebra of an arbitrary algebra $E$, and let $S, R$, be their respective representation spaces, and $\xi$ the mapping of $R$ onto $S$ introduced in 2.2. For each $x \in E$, write $x^{*}=\Lambda\{y \mid y \in A, y \geq x\}$ (the infimum referring to $E$, but $x^{*} \in A$ ). It is now easily verified that

$$
\xi R x=S x^{*},
$$

so that $\xi$ is now open as well as continuous (and so closed, as $R, S$ are compact Hausdorff). It follows that

$$
\xi^{-1} \mathscr{K} S \subset \Re R
$$

We deduce:

(3) If $H \in B^{*} S$ and $\xi^{-1} H \in \Re R$, then $H \in \Re S$.

For, by $2.1(2), H=G+Z$ where $G$ is open and $Z \in \Re S$. Hence $\xi^{-1} H$ $=\xi^{-1} G+\xi^{-1} Z$, showing that the open set $\xi^{-1} G$ is (from (2)) of first category in the compact space $R$; hence $\xi^{-1} G=\varnothing$, giving $H=Z$ as required.

These results, together with $2.2(3)$, give

$$
\nVdash R \cap \mathbb{B}_{0}=\xi^{-1}(\nVdash S \cap \mathbb{Q}) .
$$


2.4 Function spaces. Let $S$ be any set, $B$ any Borel field of subsets of $S$, and $\Re$ any $\sigma$-ideal of subsets of $S$. By a "function" $f$ on $S$ we mean an extended-real function (so that, for each $s \in S, f(s)$ is real or $\infty$ or $-\infty$ ). We make the convention that $0 \cdot \infty=0$, but $\infty-\infty$ is not defined. $F(B)$ denotes the collection of " $B$-measurable" functions on $S$, that is, of functions $f$ such that, for each real $\rho,\{s \mid s \in S, f(s)>\rho\} \in \mathbb{B}$. The set of non-negative $B$ measurable functions is denoted by $\mathfrak{F}^{+}(B)$; generally, if $Q$ is any set of functions, $a^{+}$denotes the set of non-negative functions in $a$. The support $[f]$ (called "locus" in $[4 ; 5]$ ) of a function $f$ on $S$ is the set $\{s \mid f(s) \neq 0\}$. A function $f$ is "null" or $\Re$-negligible if $[f] \in \Re$; the set of $\Re$-negligible functions is denoted by $Z(\mathscr{Z})$. The equivalence class modulo $Z(\mathscr{X})$ of a function $f$ is written $f+Z(\mathscr{I})$ or $\bar{f}$ (and not $\{f\}$, as in $[2 ; 4]$ ). We write $\bar{f} \ll \bar{g}$ to mean $f(s)<g(s)$ for all $s \in S-N$ where $N \in \mathscr{N}$, and $\bar{f} \leqq \bar{g}$ to mean $f(s) \leqq g(s), s \in S-N ; \bar{f}<\bar{g}$ means $\bar{f} \leqq \bar{g}$ and $\bar{f} \neq \bar{g}$. The set $\{\bar{f} \mid f \in \mathcal{F}(\mathbb{B})\}$ is written $\mathcal{F}(\mathbb{B}) / \mathcal{Z}(\mathfrak{T})$ or $\mathcal{F}(S, \mathbb{B}, \mathfrak{T}) .\left({ }^{4}\right)$

If $E$ is the $\sigma$-algebra $\mathbb{B} / \mathfrak{N}, E$ determines $\mathcal{F}(S, \mathbb{Q}, \mathfrak{N})$ to within "strict" isomorphism (a 1-1 correspondence preserving $\leqq$ and (pointwise) sums and products) (see $[4$, p. 159]), and we write the strict isomorphism class of $\mathcal{F}(S, \mathbb{B}, \mathfrak{N})$ as $F(E)$; we also use $F(E)$ to stand for any one member of this class. $F^{+}(E)$ of course denotes the subset of $F(E)$ corresponding to the nonnegative functions. We say that $\mathcal{F}(S, \cap, \mathfrak{N})$ is a realisation of $F(E)$. For every $\sigma$-algebra $E$, the function space $F(E)$ exists and has the representation space

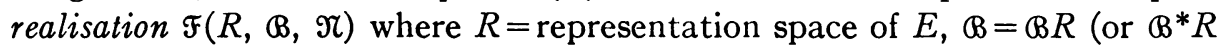
if $E$ is complete), $\Re=\varkappa R$. If $E$ is complete, each $\bar{f} \in F(E)$ now has a unique continuous representative $f \in \mathcal{F}(R, @, \Re)$, as follows from [2, p. 285].

The support $[\bar{f}]$ of $\bar{f} \in F(E)$ is the element $[f]+\mathscr{N}$ of $E$. Dually, if $x \in E$, its characteristic function $\chi_{x}$ or $\chi x$ is the element $\overline{\chi(X)}$ of $F(E)$, where $\chi(X)$ is the characteristic function of any set $X \in B$ for which the equivalence class $X+\Re=x$. The value of $\chi(X)$ at $s \in S$ is denoted by $\chi(X, s)$.

2.5 Cylinder mappings. Let $A$ be a $\sigma$-subalgebra of a $\sigma$-algebra $E$. There is then a natural strict isomorphism $c$ of $F(A)$ in $F(E)$, which we call the "cylinder mapping" (by analogy with the case in which $E$ is the product of $A$ with another factor); it can be described as follows. Each $\bar{g} \in F(A)$ determines a "spectrum" (cf. $[6 ; 4$, p. 159]) on $A$; in terms of any realisation $\mathcal{F}(S, \mathbb{B}, \mathfrak{\Re})$ of $F(A)$, the spectrum consists of the equivalence classes modulo $\mathscr{N}$ of the sets $\{s \mid g(s)<\rho\}$ where $\rho$ is rational. Conversely, each spectrum on $A$ determines a unique $\bar{g} \in F(A)$. The imbedding of $A$ in $E$ turns the spectrum of $\bar{g}$ into the spectrum of a unique $\bar{f} \in F(E)$, and we take $c(\bar{g})=\bar{f}$. In particular, if $\mathscr{F}\left(S^{\prime}, \mathbb{B}^{\prime}, \mathfrak{T}^{\prime}\right)$ is any realisation of $F(E)$, the sets of $\mathbb{B}^{\prime}$ which correspond to elements in $A$ form a Borel field $\mathbb{B}^{\prime \prime} \subset \mathbb{B}^{\prime}$ such that $\mathcal{F}\left(S^{\prime}, \mathbb{B}^{\prime \prime}, \mathfrak{T}^{\prime}\right)$ is a realisation of $F(A)$; the cylinder mapping of $F(A)$ in $F(E)$ is now that induced by the identity mapping on $S^{\prime}$.

(4) It is an "extended vector $\sigma$-lattice with a unit," in the sense that the classes of the finite functions form a vector $\sigma$-lattice with a unit, and conversely every vector $\sigma$-lattice with a unit arises in this way. 
We shall later require the form taken by the cylinder mapping in terms of the representation space realisations. Let $R, S$ be the representation spaces of the $\sigma$-algebras $E, A$ where $A$ is a complete subalgebra of $E$; let $\xi$ be the corresponding mapping of $R$ onto $S$ (cf. 2.2), and let $\mathcal{C}$, D be the families of continuous functions on $R, S$. As $\xi$ is continuous, $\xi$ induces a mapping $\xi^{*}$ of $D$ in $\mathcal{e}$ by the rule $\left(\xi^{*} g\right) p=g(\xi p), p \in R, g \in D$. Each $\bar{g} \in F(A)$ is the equivalence class of a unique $g \in D$ (cf. end of 2.4), and we have

$$
c \bar{g}=\overline{\xi^{*} g},
$$

as follows from 2.2(1) and 2.3(2) applied to the spectrum of $\bar{g}$.

$2.6 F^{\prime}$-integrals. Let $E, E^{\prime}$ be $\sigma$-algebras satisfying the countable chain condition, and write $F=F(E), F^{\prime}=F\left(E^{\prime}\right)$. A mapping $\phi$ of a subset $G$ of $F$ in $F^{\prime}$ is called a positive linear operator from $F$ to $F^{\prime}$, or an $F^{\prime}$-integral on $F$ (cf. $[4$, p. $161 ; 5$, p. 232$])$ if $G \supset F^{+}$and:

(1) If $\bar{f} \in F^{+}, \phi \bar{f} \geqq 0$.

(2) If $\bar{f}_{n} \in F^{+}(n=1,2, \cdots)$, then $\phi\left(\sum \bar{f}_{n}\right)=\sum \phi \bar{f}_{n}$.

(3) There exist $\bar{g}_{1}, \bar{g}_{2}, \cdots \in F^{+}$such that $\sum \bar{g}_{n} \gg 0$ and $\phi \bar{g}_{n} \ll \infty$.

(4) $G=\left\{\bar{f} \mid \phi\left(\bar{f}^{+}\right) \wedge \phi\left(\bar{f}^{-}\right) \ll \infty\right\}$, and if $\bar{f} \in G$ then $\phi \bar{f}=\phi\left(\bar{f}^{+}\right)-\phi\left(\bar{f}^{-}\right)$.

As $\phi$ is determined by its values on $F^{+}$, we shall usually regard $\phi$ as a mapping of $F^{+}$in $F^{\prime+}$ satisfying (2) and (3); for every such mapping can be extended to a suitable $G[4$, pp. 161,162$]$. The extended mapping $\phi$ is necessarily linear on $G$.

If further $\phi$ satisfies

(i) $\phi \bar{f}>0$, if $\bar{f}>0$,

(ii) $\phi 1 \gg 0$,

$\phi$ is called strict. (In $[4 ; 5]$, a strict $F^{\prime}$-integral on $F$ was called simply an $F^{\prime}$ integral; the present $F^{\prime}$-integral was called "relaxed.") Every $F^{\prime}$-integral $\phi$ on $F$ determines in a natural way a strict $F_{1}^{\prime}$-integral $\phi_{1}$ on $F_{1}$, where $F_{1}=F\left(E_{1}\right)$, $F_{1}^{\prime}=F\left(E_{1}^{\prime}\right)$, and $E_{1}, E_{1}^{\prime}$ are suitable principal ideals of $E, E^{\prime}$ (see [5, p. 238]); $\phi_{1}$ is called the "strict form" of $\phi$.

For any $F^{\prime}$-integral $\phi$ on $F$, we write $\lambda x=\phi(\chi x)(x \in E) ; \lambda$ is the induced " $F^{\prime}$-measure" on $E$; it is countably additive and $\sigma$-finite $[5$, p. 233 (a) and (b)] and determines $\phi$ uniquely.

An $F^{\prime}$-integral $\phi$ on $F$ is fully homogeneous if the corresponding $F$-measure $\lambda$ is "full-valued" in the sense of $\left[4\right.$, p. 166]; that is, given $x \in E$ and $\bar{g} \in F^{\prime+}$ such that $\bar{g} \leqq \lambda x$, there exists $y \leqq x$ such that $\lambda y=\bar{g}$. A fully homogeneous $\phi$ is itself full-valued $[4$, p. 174$]$; that is, given $\bar{f} \in F^{+}$and $\bar{g} \in F^{\prime+}$ such that $\bar{g} \leqq \phi \bar{f}$, there exists $\bar{h} \in F^{+}$such that $\bar{h} \leqq \bar{f}$ and $\phi \bar{h}=\bar{g}$.

Let $E, E^{\prime}, E^{\prime \prime}$ be $\sigma$-algebras, and $F, F^{\prime}, F^{\prime \prime}$ their function spaces (that is, $F^{\prime}=F\left(E^{\prime}\right)$, etc.). Suppose $\phi$ is an $F^{\prime}$-integral on $F$, and $\psi$ an $F^{\prime \prime}$-integral on $F^{\prime}$. In general, $\psi \phi$ need not be an $F^{\prime \prime}$-integral on $F$, as the $\sigma$-finiteness requirement (3) may fail. But:

(5) If $\phi$ and $\psi$ are fully homogeneous, $\psi \phi$ is a fully homogeneous $F^{\prime \prime}$ - 
integral on $F$, provided that $E$ satisfies the countable chain condition.

There is no difficulty in seeing that $\psi \phi$ satisfies conditions (1) and (2). Suppose $0 \leqq g \leqq \psi \phi \chi x$ where $x \in E, g \in F^{\prime \prime}$. Put $h=\lambda x=\phi \chi x$; then $0 \leqq g \leqq \psi h$, so (as $\psi$ is full-valued) there exists $k \in F^{\prime}$ such that $k \leqq h$ and $\psi k=g$. Hence there exists $y \leqq x$ in $E$ such that $\lambda y=k$; and $\psi \phi \chi y=g$, proving that $\psi \phi$ is fully homogeneous. Put $\psi \phi=\theta$; condition (3) now follows in this way. Put $h_{1}=1 \wedge \theta 1$; there exists $x_{1} \in E$ such that $\theta \chi x_{1}=h_{1}$. If $\alpha$ is any countable ordinal, and disjoint elements $x_{\beta} \in E$ have been defined for all $\beta<\alpha$ so that $\theta \chi x \leqq 1$, we put $h_{\alpha}=1 \wedge \theta \chi\left(-\bigvee x_{\beta} \mid \beta<\alpha\right)$. If $h_{\alpha} \neq 0$, we take $x_{\alpha} \leqq-\bigvee x_{\beta}$ so that $\theta \chi x_{\alpha}=h_{\alpha} \leqq 1$; if $h_{\alpha}=0$, we put $x_{\alpha}=-V x_{\beta}$ and terminate the process. Because of the countable chain condition, this process terminates for some countable $\alpha$. Renumbering the elements $x_{\beta}(\beta<\alpha)$ into a simple sequence $x_{1}, \cdots, x_{n}, \cdots$, we put $\bar{g}_{n}=\chi x_{n}$ and have $\sum \bar{g}_{n}=1 \gg 0$ and $\theta \bar{g}_{n} \leqq 1 \ll \infty$ as required.

For any $F^{\prime}$-integral $\phi$ on $F$ we have:

(6) If $f, g \in F^{+}$and $[f]=[g]$, then $[\phi f]=[\phi g]$.

For $\infty \cdot f=\infty \cdot g$; hence $[\phi f]=[\infty \phi f]=[\phi \infty f]=[\phi g]$.

Now let $E_{1}, E_{1}^{\prime}$ be $\sigma$-subalgebras of $\sigma$-algebras $E_{2}, E_{2}^{\prime}$ satisfying the countable chain condition; write $F_{i}, F_{i}^{\prime}$ for $F\left(E_{i}\right), F\left(E_{i}^{\prime}\right)$ respectively $(i=1,2)$, and let $c, c^{\prime}$ be the respective cylinder mappings of $F_{1}$ in $F_{2}, F_{1}^{\prime}$ in $F_{2}^{\prime}$. If $\phi_{i}(i=1,2)$ is an $F_{i}^{\prime}$-integral on $F_{i}$ we say that $\phi_{2}$ is a cylinder extension of $\phi_{1}$ if, for each $\bar{f} \in F_{1}^{+}, c^{\prime}\left(\phi_{1} \bar{f}\right)=\phi_{2}(c \bar{f})$. As remarked in 2.5, we can choose realisations of $F_{i}$ and $F_{i}^{\prime}$ for which $c^{\prime}$ and $c$ are induced by identity mappings, and then $\phi_{2}$ is a cylinder extension of $\phi_{1}$ if and only if $\phi_{1}$ is (in an obvious sense) the restriction of $\phi_{2}$ to $F_{1}$.

The following result is basic for the construction in the present paper. It is proved (in a slightly different formulation) in [4, Theorem 6] for the case in which $\phi$ is strict; the general result follows easily on considering the "strict form" of $\phi$. (For details see the beginning of $\$ 7$.)

(7) If $\phi$ is an $F^{\prime}$-integral on $F$, where $F=F(E)$ and $F^{\prime}=F\left(E^{\prime}\right)$, there exists an algebra $E^{*}$, of which $E$ is a complete subalgebra, and an $F^{\prime}$-integral $\phi^{*}$ on $F(E)$, such that $\phi^{*}$ is a fully homogeneous cylinder extension of $\phi$.

Except where the contrary is stated, all algebras in what follows are assumed to be $\sigma$-algebras satisfying the countable chain condition. Further, the term "subalgebra" is understood to mean a $\sigma$-subalgebra, and hence a complete subalgebra.

\section{Functions on representation spaces.}

3.1 Let $A$ be a (complete) subalgebra of an arbitrary algebra $E$; $A$ is of course assumed to be a $\sigma$-algebra satisfying the countable chain condition, but $E$ is not. We derive for later use some properties of the representation spaces $R, S$ of $E, A$, and of various families of functions on them. We write $\mathfrak{T}_{0}^{\prime}=B_{0} \cap \mathcal{K} R$, where $B_{0}$ is the Borel field generated by the sets $R y, y \in A$, and $\Re_{0}$ for the family of all subsets $N$ of $R$ which are subsets of sets in $\Re_{0}^{\prime}$. From 2.2 and $2.3(4), \mathcal{F}\left(R, \bigotimes_{0}, \Re_{0}\right)$ and $\mathcal{F}\left(R, \bigotimes_{0}, \Re R\right)$ are realisations of $F(A)$; if $E$ 
is a $\sigma$-algebra, the corresponding cylinder mappings of $F(A)$ in $F(E)$ are induced by the identity mapping. As in 2.2 we write $\xi$ for the natural mapping of $R$ onto $S$, and $\mathfrak{C}, \mathbb{D}$ for the families of continuous functions on $R, S$.

3.2 Lemma. If $H \in \Re S$, there exist $x_{m n} \in A(m, n=1,2, \cdots)$ such that $x_{m 1} \geqq x_{m 2} \geqq \cdots, \Lambda_{n} x_{m n}=0$ for each $m$, and $H \subset \bigcup_{m} \cap_{n} S x_{m n}$.

For let $F$ be closed and nowhere dense in $S$; the open set $S-F$ can be written as $U S y_{\lambda}$ for suitable $y_{\lambda} \in A$, and (because $F$ is nowhere dense) $V y_{\lambda}=e$. There is therefore a sequence of values of $\lambda$, which we denote by $1,2, \cdots$, $i, \cdots$, such that $\bigvee y_{i}=e$. Put $x_{n}=-\left(y_{1} \bigvee y_{2} \bigvee \cdots \bigvee y_{n}\right)$; then $x_{1} \geqq x_{2} \geqq \cdots$, $\Lambda x_{n}=o, \quad$ and $S x_{n}=S-\left(S y_{1} \cup \cdots \cup S y_{n}\right) \supset F$. Now we have $H \subset \cup F_{m}(m=1,2, \cdots)$ where $F_{m}$ is closed and nowhere dense; applying the foregoing to $F_{m}$ instead of $F$, we obtain the elements $x_{m n}$ required.

Corollary. If $H \in \Re S$, there exists $H^{*} \in \varkappa S \cap @ S$ such that $H \subset H^{*}$.

3.3 Definition. A function $f$ on $R$ is 0 -continuous if, for each real (or, equivalently, rational) $\rho,\{p \mid p \in R, f(p)>\rho\}$ is a union of sets of the form $R y$ where $y \in A$. (This implies that $f$ is continuous.) The set of 0 -continuous functions on $R$ is denoted by $\mathfrak{C}_{0}$. We have

(1) $f \in \mathcal{F}\left(\mathbb{B}_{0}\right)$ if and only if there exists $h \in \mathcal{F}(\mathbb{B}(S))$ such that $f=h \xi$.

The nontrivial implication here can be seen by considering the spectrum of $f$, or by observing that (by the argument in $\left[4\right.$, p. 157]) each $f \in \mathcal{F}\left(\mathbb{B}_{0}\right)$ is expressible as $\sum \alpha_{n} \chi X_{n}$ where $X_{n} \in \Theta_{0}$ and $\alpha_{n}$ is real. We have $X_{n}=\xi^{-1} Y_{n}$ where $Y_{n} \in ß S$, and then, if we set $h=\sum \alpha_{n} \chi Y_{n}$, we have $f=h \xi$.

We deduce:

(2) If $f \in \mathcal{F}\left(\bigotimes_{0}\right)$, there exists $h \in \mathfrak{C}_{0}$ such that $f=h \bmod \mathfrak{N}_{0}$.

By (1), $f=g \xi$ where $g \in \mathcal{F}(\circledast(S))$. There exists a continuous function $g_{1}$ on $S$ such that $g(s)=g_{1}(s)$ for $s \in S-H$, where $H \in \Re S$; and by 3.2, Corollary, we may assume $H \in \varnothing S$ also. Put $h=g_{1} \xi$. Using $2.2(1)$ we see that $h$ is 0 continuous; and $f(p)=h(p)$ for $p \in R-N$ where $N=\xi^{-1} H \in \Re_{0}$ by $2.3(4)$. Conversely:

(3) If $f \in \mathfrak{C}_{0}$, there exists $g \in \mathcal{F}\left(\Theta_{0}\right)$ such that $f=g \bmod \mathfrak{T}_{0}$.

For write $X_{p}=\{p \mid p \in R, f(p)<p\}$; by hypothesis, this is of the form $\cup R y_{\alpha}$ for suitable elements $y_{\alpha} \in A$. Let $z_{\rho}=\bigvee y_{\alpha}$; then $R z_{\rho}=X_{\rho} \cup H_{\rho}$ where $H_{\rho}=\xi^{-1}\left(S z_{\rho}-\cup S y_{\alpha}\right)$. As $S z_{\rho}-U S y_{\alpha}$ is closed and nowhere dense in $S$, it is contained in a set $K_{\rho} \in \mathcal{K} S \cap \propto S$ (3.2, Corollary); hence $H_{\rho} \subset \xi^{-1} K_{\rho} \in \mathfrak{\Re}_{0}$. Put $N=\bigcup\left\{\xi^{-1} K_{\rho} \mid \rho\right.$ rational $\}, g=f \chi(R-N)$. Then $N \in \mathfrak{N}_{0}$, and $f(p)=g(p)$ for $p \in R-N$. Let $Y_{\rho}=\{p \mid p \in R, g(p)<\rho\}$; one verifies that, if $\rho$ is rational, $Y_{\rho}=R z_{\rho} \cap(R-N)$ for $\rho \leqq 0, Y_{\rho}=R z_{\rho} \cup N$ for $\rho>0$. Hence $Y_{\rho} \in Q_{0}$ for all rational $\rho$, and hence for all $\rho$, proving $g \in F\left(B_{0}\right)$.

Next we deduce:

(4) $f \in \mathfrak{C}_{0}$ if and only if $f=h \xi$ for some $h \in \mathcal{D}$.

The "if" is trivial from 2.2(1). Conversely, given $f \in \mathfrak{C}_{0}$, apply (3) and (1) 
to obtain $g \in \mathcal{F}(B(S))$ such that $f=g \xi$ modulo $\Re_{0}$. There exists a continuous $h$ on $S$ such that $h(s)=g(s)$ for $s \in S-H$, where $H \in \Re S$. Thus $f(p)=h \xi(p)$ for all $p \in R-N$ where $N \in \mathcal{K} R$. As $R-N$ is dense in $R$, and $f, h \xi$ are both continuous, it follows that $f(p)=h \xi(p)$ for all $p \in R$.

3.4 Let $\mathfrak{T}_{0}$ denote $\beta_{0}+\mathscr{N}_{0}$ (that is, the family of all sets of the form $B+N$ where $B \in \Re_{0}, N \in \Re_{0}$ ). Equivalently (from 2.1(2), 2.2(3), 2.3(4)) $\mathfrak{M}_{0}$ consists of all sets of the form $R y+N$ where $y \in A, N \in \Re_{0}$. Clearly $\mathfrak{M}_{0}$ is a Borel field. We say that a function $f$ on $R$ is 0 -measurable if all the sets $\{p \mid f(p)<\rho\}$ are in $\mathfrak{T}_{0}$. If $f \in Z \mathcal{Z}\left(\mathscr{N}_{0}\right)$ (that is, if $[f] \in \mathscr{N}_{0}$ ) we say that $f$ is 0 negligible. Every 0 -continuous function is 0 -measurable (cf. end of 2.1). Conversely,

(1) Given an 0 -measurable function $f$, there exists a unique 0 -continuous function $g$ such that $f=g \bmod \mathfrak{\Re}_{0}$.

By $\left[4\right.$, p. 157] we have $f=\sum \alpha_{n} \chi X_{n}$ for suitable real numbers $\alpha_{n}$ and sets $X_{n} \in \mathfrak{M}_{0}(n=1,2, \cdots)$; and each $X_{n}$ has the form $R x_{n}+H_{n}$ where $H_{n} \in \Re_{0}$ and $x_{n} \in A$. Consider the function $h=\sum \alpha_{n} \chi S x_{n}$ on $S$; being $B S$-measurable, it differs from a continuous function $k$ on $S$ on a first category set $K$. Then $g=k \xi$ is 0 -continuous, and we have $f(p)=k \xi(p)$ if $p \in R-\left\{\xi^{-1} K \cup \cup H_{n}\right\}$. The uniqueness of $\mathrm{g}$ is trivial.

Given $\bar{g} \in F(A)$, the class $\bar{g}$, in the representation space realisation of $F(A)$, contains a unique continuous function $g_{0}\left[2\right.$, p. 287]. Then $g_{0} \xi$ is 0 continuous on $R$; further, from 3.3(4), every 0 -continuous function arises in this way. We write $g_{0} \xi=R_{0} \bar{g} ; R_{0}$ induces a strict isomorphism (a 1-1 correspondence preserving $\leqq$ and finite sums and products) between $F(A)$ and $\mathfrak{C}_{0}$. It follows that every subset $\left\{f_{\alpha}\right\}$ of $\mathfrak{C}_{0}$ has a least upper bound $f=\bigvee f_{\alpha}$ in $\mathfrak{C}_{0}$, and there is a countable subfamily $\left\{f_{\alpha_{n}}\right\}$ of $\left\{f_{\alpha}\right\}$ such that $f=\bigvee f_{\alpha_{n}}$. Moreover we have

(2) If $f=\bigvee f_{\alpha}$ in $\mathfrak{C}_{0}, f(p)=\sup f_{\alpha}(p)$ for all $p \in R-N$, where $N \in \mathfrak{Y}_{0}$.

For we have $f_{\alpha}=g_{\alpha} \xi$ where $g_{\alpha}$ is continuous on $S$, and if $\bar{g}=\bigvee_{\bar{g}_{\alpha}}$ in $F(A)$ then $\bar{g}=\bigvee \bar{g}_{\alpha_{n}}$. If $g_{0}$ is the continuous function on $S$ which is in $\bar{g}$, then $g_{0}(s)$ $=\sup g_{\alpha_{n}}(s)$ if $s \in S-H$ where $H \in \mathcal{K} S$, and we can assume (3.2) $H \in \mathbb{B} S$ also. Then $f=g_{0} \xi$, and $f(p)=\sup f_{\alpha}(p)$ if $p \in R-\xi^{-1} H$ where $\xi^{-1} H \in \Re_{0}$ by $2.3(4)$.

Note that, from 2.2(1),

$$
R_{0} \chi y=\chi R y, \quad \text { if } y \in A .
$$

If $E$ is itself a $\sigma$-algebra, the cylinder mapping $c$ of $F(A)$ in $F(E)$ is defined, and from $2.5(1)$ we have, for $\bar{g} \in F(A)$,

(4) $R_{0} \vec{g}$ is the unique 0 -continuous function in the class $\left.c \bar{g} .{ }^{5}\right)$

For later use, we deduce:

$\left(^{(5)}\right.$ Even when $E$ is only finitely additive, it would be possible to define a "cylinder mapping" from $F(A)$ to the finitely additive function space corresponding to $F(E)$, whenever $A$ is a $\sigma$-subalgebra of $E$. This mapping would then be realised by $R_{0}$. 
(5) If $f \in \mathfrak{C}_{0}^{+}$, there exist positive real numbers $\sigma_{n}$, elements $x_{n} \in A$ $(n=1,2, \cdots)$, and a non-negative function $h$ such that

$$
f=\sum \sigma_{n} \chi R x_{n}+h .
$$

We have $f=R_{0} \bar{g}$ where $\bar{g} \in F(A)^{+}$, and a slight modification of the argument in $\left[4\right.$, p. 157, Lemma 1] gives $\bar{g}=\sum \sigma_{n} \chi x_{n}$ where $x_{n} \in A$ and $\sigma_{n}>0$. Hence, by (3), $f=\sum \sigma_{n} \chi R x_{n}$ on $R-N$, where $N \in \mathfrak{N}_{0}$. By continuity, $f \geqq$ every finite sum of terms $\sigma_{n} \chi R x_{n}$, so the difference $h$ between $f$ and $\sum \sigma_{n} \chi R x_{n}$ is $\geqq 0$.

4. The main theorem and its proof (first part).

4.1 We now state the main theorem of this paper. We recall that "algebra" means "Boolean $\sigma$-algebra satisfying the countable chain condition" except where the contrary is stated.

TheOREM 1. Let $E_{0}$ be an algebra, $F_{0}=F\left(E_{0}\right)$ its function space, and $\phi_{0} a$ positive linear operator from $F_{0}$ to itself (that is, an $F_{0}$-integral on $F_{0}$ ). There exist an algebra $E$, of which $E_{0}$ is a subalgebra, and a fully homogeneous $F$ integral $\phi$ on $F$, where $F=F(E)$, such that $\phi$ is a cylinder extension of $\phi_{0}$.

REMARK. If $\phi_{0}$ is a strict $F_{0}$-integral on $F_{0}$, I do not know whether $\phi$ can always be taken to be a strict $F$-integral on $F$. If $\phi_{0}(1) \gg 0$, then automatically $\phi(1) \gg 0$ because $\phi$ is a cylinder extension.

Before proving the theorem, we note the following consequence of it.

Corollary. For each $n=1,2, \cdots, \phi^{n}$ is also a fully homogeneous $F$ integral on $F$, and is a cylinder extension of $\phi_{0}^{n}$.

This follows from Theorem 1 by an easy induction, using 2.6(5).

4.2 The algebras $E_{n}$. The proof of Theorem 1 requires a number of steps. First we note that by successive applications of 2.6(7) we obtain a sequence of algebras $E_{0}, E_{1}, E_{2}, \cdots$, where $E_{k}$ is a subalgebra of $E_{k+1}$, and a sequence $\left\{\phi_{k}\right\}(k=0,1,2, \cdots)$ where $\phi_{k}$ is an $F_{k}$-integral on $F_{k}, F_{k}$ denoting $F\left(E_{k}\right)$, such that

(1) $\phi_{k+1}$ is a cylinder extension of $\phi_{k}$; that is, $\phi_{k+1} c_{k, k+1}=c_{k, k+1} \phi_{k}$, where $c_{k, k+1}$ is the cylinder mapping of $F_{k}$ in $F_{k+1}$,

(2) $\phi_{k+1}\left(F_{k+1}^{+}\right) \subset c_{k, k+1}\left(F_{k}^{+}\right)$, and further

(3) $\left(c_{k, k+1}\right)^{-1} \phi_{k+1}$ is a fully homogeneous $F_{k}$-integral on $F_{k+1}$.

(We merely put $\phi_{k+1}=c_{k, k+1} \phi^{*}$ where $\phi^{*}$ is the extension provided by 2.6(7).)

We write $c_{n, n+k}$ for the cylinder mapping of $F_{n}$ into $F_{n+k}(n, k \geqq 0)$, noting that $c_{n, n+k}$ is $1-1$, that $c_{n n}$ is the identity mapping, and that $c_{n, n+h+k}$ $=c_{n+h, n+h+k} c_{n, n+h}$. To simplify printing, we write the inverse mapping $\left(c_{n, n+k}\right)^{-1}$ as $c_{n+k, n}$. By induction, first over $k$ for $m=1$ and then over $m$, we obtain

$$
\left(\phi_{n+k}\right)^{m} c_{n, n+k}=c_{n, n+k}\left(\phi_{n}\right)^{m} \text { on } F_{n}^{+} \quad(m=1,2, \cdots) .
$$

Restated in terms of the inverse cylinder mappings, this is 


$$
c_{n+k, n}\left(\phi_{n+k}\right)^{m}=\left(\phi_{n}\right)^{m} c_{n+k, n} \text { on } c_{n, n+k} F_{n}^{+} \subset F_{n+k}^{+} .
$$

Next we show

$$
\begin{aligned}
\left(\phi_{n+k}\right)^{k} F_{n+k}^{+} \subset c_{n, n+k} F_{n}^{+}, \text {and } & c_{n+k, n}\left(\phi_{n+k}\right)^{k} \text { is a fully } \\
& \text { homogeneous } F_{n} \text {-integral on } F_{n+k} .
\end{aligned}
$$

In fact, if $r \geqq 1, c_{n+r, n+r-1} \phi_{n+r}$ is a fully homogeneous $F_{n+r-1}$-integral on $F_{n+r}$, by (3). Now, on $F_{n+k}^{+}$, put

$$
\psi=\left(c_{n+1, n} \phi_{n+1}\right)\left(c_{n+2, n+1} \phi_{n+2}\right) \cdots\left(c_{n+k, n+k-1} \phi_{n+k}\right) .
$$

Then $\psi$ is a fully homogeneous $F_{n}$-integral on $F_{n+k}$, by 2.6(5). But, in view of (5),

$$
\begin{aligned}
\psi & =c_{n+1, n}\left(\phi_{n+1} c_{n+2, n+1}\right) \cdots\left(\phi_{n+k-1} c_{n+k, n+k-1}\right) \phi_{n+k} \\
& =c_{n+1, n} c_{n+2, n+1} \phi_{n+2} \phi_{n+2} c_{n+3, n+2} \cdots \\
& =c_{n+2, n}\left(\phi_{n+2}\right)^{2} c_{n+3, n+2} \phi_{n+3} \cdots \\
& =c_{n+2, n} c_{n+3, n+2}\left(\phi_{n+3}\right)^{3} \cdots=c_{n+k, n}\left(\phi_{n+k}\right)^{k} \text { finally. }
\end{aligned}
$$

This also shows $c_{n+k, n}\left(\phi_{n+k}\right)^{k}$ is defined for all $\bar{f} \in F_{n+k}^{+}$, giving the first part of the assertion.

REMARK. It follows from (6) that $\left(\phi_{n}\right)^{m}$ is an $F_{n}$-integral on $F_{n}$ provided $m \leqq n$; compare footnote 3 .

4.3 The algebra $E^{\prime}$. Let $E^{\prime}=\bigcup E_{n}$, where $E_{0}, E_{1}, E_{2}, \cdots$ is the sequence of algebras obtained in 4.2. For $x, y \in E^{\prime}$, define $x \leqq y$ to mean that $x \leqq y$ in some $E_{n}$ (and so in $E_{m}$ for all $m \geqq n$ ). It is easily verified that $E^{\prime}$ becomes a finitely additive Boolean algebra satisfying the countable chain condition, and that each $E_{n}$ is a complete subalgebra of $E^{\prime}$. Let $R$ be the representation space of $E^{\prime}$. The required extended function space $F$ of Theorem 1 (4.1) will be defined by a certain realisation $F(R, B, \Re)$; but before we define $B$ and $\Re$ it is convenient to have the extended integral $\phi$ more or less available. This we achieve by defining an operator $\Phi$ on a suitable class $\mathbb{C}^{\prime}$ of continuous functions on $R$ (4.5). By measure-theoretic considerations we are then able to extend a modified form of $\Phi$ to an operator $\Phi^{*}$ on the family $\mathcal{F}\left(\mathfrak{N}^{\prime}\right)$ of $\mathfrak{M}^{\prime}$ measurable functions, where $\mathfrak{T}^{\prime}$ is a certain Borel field of subsets of $R(4.8)$; and all that remains is to define the ideal $\Re$ of null sets-an operation of some delicacy since $\Re$ must be large enough to produce the countable chain condition and not so large as to annihilate $\Phi^{*}$.

4.4 The function-class $\mathrm{C}^{\prime}$. As in $\$ 2$, we let $R x$ denote the open-closed subset of $R$ corresponding to $x \in E^{\prime}$. We write $\mathcal{E}^{\prime}=\left\{R x \mid x \in E^{\prime}\right\}, \varepsilon_{k}=\left\{R x \mid x \in E_{k}\right\}$, $\mathbb{B}^{\prime}=\mathbb{B} R=$ Borel field (of subsets of $R$ ) generated by $\mathcal{E}^{\prime}, \mathbb{B}_{k}=$ Borel field generated by $\varepsilon_{k}$. A set $N \subset R$ is called $k$-negligible if it is contained in some $Y \in$ $\mathbb{B}_{k} \cap \varkappa R ; N$ is negligible if it is of the form $U N_{k}(k=0,1, \cdots)$ where $N_{k}$ is $k$ negligible. The families of $k$-negligible and of negligible sets are written 
$\mathfrak{N}_{k}, \mathfrak{N}^{\prime}$ respectively; they are $\sigma$-ideals. We have $\mathbb{B}_{0} \subset \bigotimes_{1} \subset \cdots \subset \mathbb{B}^{\prime}, \mathfrak{N}_{0} \subset \mathfrak{N}_{1}$ $\subset \cdots \subset \mathfrak{N}^{\prime} \subset \Re R$. We say that a function on $R$ is $k$-negligible or negligible if it is in $Z\left(\mathscr{N}_{k}\right)$ or $Z\left(\mathscr{N}^{\prime}\right)$, respectively.

We define $\mathfrak{T}_{k}=\mathfrak{B}_{k}+\mathfrak{N}_{k}$ (cf. 3.4), $\mathfrak{N}_{k}^{\prime}=\mathfrak{B}_{k}+\mathfrak{N}^{\prime}, \mathfrak{T}^{\prime}=\mathbb{B}^{\prime}+\mathfrak{N}^{\prime}$; all these are Borel fields. By 3.3(2), $B_{k} \subset \mathcal{E}_{k}+\mathfrak{T}_{k}$, so that $\mathfrak{T}_{k}=\mathcal{E}_{k}+\mathfrak{N}_{k}$, and hence $\mathfrak{T}_{k}^{\prime}=$ $\mathcal{E}_{k}+\mathfrak{N}^{\prime}$. Clearly $\mathfrak{M}_{0} \subset \mathfrak{M}_{1} \subset \cdots, \mathfrak{M}_{0}^{\prime} \subset \mathfrak{M}_{1}^{\prime} \subset \cdots$, and $\mathfrak{T}_{k} \subset \mathfrak{M}_{k}^{\prime} \subset \mathfrak{M T}^{\prime}$. If $x \in E_{k}$, the correspondence between $x$ and $(R x)+\mathfrak{T}_{k}$ is a (complete) isomorphism between $E_{k}$ and $\mathfrak{T}_{k} / \mathfrak{T}_{k}$; similarly, for $x \in E^{\prime}$, the correspondence between $x$ and $(R x)+\mathscr{N}^{\prime}$ is a finite isomorphism between $E^{\prime}$ and a finitely additive subalgebra of $\mathfrak{T}^{\prime} / \mathfrak{T}^{\prime}$. (Note that in general $E^{\prime}$ need not be a $\sigma$ algebra, and that the $\sigma$-algebra $\mathfrak{T l}^{\prime} / \mathfrak{N}^{\prime}$ need not satisfy the countable chain condition.) If we restrict $x$ to $E_{k}$ here, we obtain an isomorphism of $E_{k}$ onto the (complete) subalgebra $\mathfrak{T}_{k}^{\prime} / \mathfrak{N}^{\prime}$ of $\mathfrak{T}^{\prime} / \mathfrak{N}^{\prime}$.

We call a function $f$ on $R$ " $k$-continuous" if for each real $\rho\{p \mid f(p)>\rho\}$ is a union of sets in $\mathcal{E}_{k}$; that is, if $f$ is " 0 -continuous" in the sense of 3.3 , taking $E=E^{\prime}, A=E_{k}$. We write $\mathfrak{C}_{k}$ for the family of all $k$-continuous functions on $R$, and $\mathfrak{e}^{\prime}$ for $\cup \mathfrak{e}_{k}$; note that $\mathfrak{e}_{0} \subset \mathfrak{e}_{1} \subset \ldots$. A function is called " $k$-measurable" if it is in $\mathscr{F}\left(\mathscr{T}_{k}\right)$ (this notation is consistent with that in 3.4$)$, " $k^{\prime}$-measurable" if it is in $\mathcal{F}\left(\mathfrak{M}_{k}^{\prime}\right)$. The following assertions follow easily from 3.3(3) and 3.4(1):

(1) Every $k$-continuous function is $k$-measurable, and hence $k^{\prime}$-measurable.

(2) If $f$ is $k$-measurable, there is a unique $k$-continuous function $g_{k}$ such that $f=g_{k} \bmod \mathfrak{N}_{k}$; and $g_{k}=g_{k+1}=\ldots$.

Let $R_{k}$ be the isomorphism between $F_{k}=F\left(E_{k}\right)$ and $\mathfrak{C}_{k}$ described in 3.4 (where we replace $A$ by $E_{k}, E$ by $E^{\prime}$ and $R_{0}$ by $R_{k}$ ).( $\left.{ }^{6}\right)$ If we use the realisation $\mathfrak{F}\left(R, \mathfrak{T}_{k}^{\prime}, \mathfrak{T}^{\prime}\right)$ of $F_{k}$, a typical element $\bar{f}$ of $F_{k}$ consists of all functions differing from a $k^{\prime}$-measurable function $f$ by a negligible function, the cylinder mapping $c_{k, k+n}$ becomes the identity mapping, and $R_{k} \bar{f}$ is the unique $k$-continuous function in $\bar{f}$. It follows that, for arbitrary realisations,

$$
R_{k} \bar{f}=R_{k+n} c_{k, k+n} \bar{f} \quad\left(k, n=0,1,2, \cdots, \bar{f} \in F_{k}\right) .
$$

4.5 The operator $\Phi$. Given $f \in \mathcal{C}^{\prime+}$, we have $f \in \mathcal{C}_{k}$ for some $k$; put $\bar{g}=R_{\mathbf{k}}^{-1} f$ $\in F_{k}$, and define $\Phi f=R_{k} \phi_{k} \bar{g} \in \mathcal{C}_{k}^{+}$. This definition does not depend on the choice of $k$, as follows from 4.4(3) and 4.2(4), so $\Phi$ is a single-valued mapping of $\mathfrak{e}^{\prime+}$ into itself. $\Phi$ and its iterates have the following properties; in all of them, $k=0,1,2, \cdots, m, n=1,2, \cdots$, and, where no proofs are given, the proofs are straightforward inductions over $m$.

(1) If $f=R_{k} \bar{g}$, where $\bar{g} \in F_{\mathbf{k}}^{+}$, then $\Phi^{m} f=R_{k} \phi_{k}^{m} \bar{g}$.

(2) $\Phi^{m}\left(C_{\boldsymbol{k}+m}^{+}\right) \subset C_{\boldsymbol{k}}^{+}$.

The case $m=1$ of (2) follows from the definition of $\Phi$, together with 4.2(2) and $4.4(3)$; the general case then follows by induction over $m$.

(6) Our notation in this paragraph is not quite exact; some isomorphisms have been suppressed. Strictly speaking, $R_{k}$ depends on which realisation of $F_{k}$ is used, but this should not cause confusion here. 
(3) If $f, g \in \mathfrak{C}^{\prime+}$ and $a, b$ are non-negative real numbers,

$$
\Phi^{m}(a f+b g)=a \Phi^{m} f+b \Phi^{m} g .
$$

(4) Let $f_{n}, f \in \mathcal{C}_{\boldsymbol{k}}^{+}$, and write $\Phi^{m} f_{n}=g_{n}, \Phi^{m} f=g$. Suppose that $f_{n}(p) \rightarrow f(p)$ for each $p \in R-N$, where $N \in \Re_{k}$, and that either (a) $f_{1} \leqq f_{2} \leqq \cdots$ or (b) $f_{1} \geqq f_{2} \geqq \cdots$ and $g_{1}(p)<\infty$ if $p \in R-N$. Then $g_{n}(p) \rightarrow g(p)$ for all $p \in R-N^{*}$ where $N^{*} \in \mathfrak{I}_{k}$.

(5) If $X \in \mathcal{E}_{k+m}, g \in \mathcal{C}_{\boldsymbol{k}}^{+}$, and $g \leqq \Phi^{m} \chi X$, then $g=\Phi^{m} \chi Y$ for some $Y \in \mathcal{E}_{k+m}$.

Here induction is not needed for the proof, which is a straightforward calculation based on 4.2(6).

As an immediate consequence of $(5), \Phi^{m}$ is "fully homogeneous" in the following sense:

(6) If $X \in \mathcal{E}^{\prime}, g \in \mathcal{C}^{++}$, and $g \leqq \Phi^{m} \chi X$, then $g=\Phi^{m} \chi Y$ for some $Y \in \mathcal{E}^{\prime}$.

Finally $\Phi^{m}$ has the following $\sigma$-finiteness property:

(7) Given $k(=0,1,2, \cdots)$, there exist disjoint sets $G_{n} \in \mathcal{E}_{k+1}(n=1,2, \cdots)$ such that $R-\cup G_{n} \in \Re_{k+1}$ and $\Phi^{k} \chi G_{n} \leqq 1$.

For, by $4.2(6), c_{k+1,1}\left(\phi_{k+1}\right)^{k}$ is a fully homogeneous $F_{1}$-integral on $F_{k+1}$. The argument proving 2.6(5) shows that elements $y_{1}, y_{2}, \cdots$ exist in $E_{k+1}$ such that $\mathrm{V} y_{n}=e$ and $\phi_{k+1}^{k} \chi y_{n} \leqq 1$. We take $G_{n}=R y_{n}$; then $G_{n} \in \mathcal{E}_{k+1}, R-U G_{n}$ is $(k+1)$-negligible, and finally, by (1) above, $\Phi^{k} \chi G_{n}=R_{k+1} \phi_{k+1}^{k} \chi y_{n} \leqq 1$.

4.6 The measures $\mu_{p}$ and $\nu_{p}$. Using 4.5(7), we take a sequence of disjoint sets $G_{n} \in \mathcal{E}_{1}$ such that $R-\cup G_{n}=Z \in \mathscr{F}_{1}$ and $\Phi \chi G_{n} \leqq 1$; these sets will remain fixed throughout the rest of the proof. Let $p$ be any point of $R$, fixed for the moment. For each $X \in \mathcal{E}^{\prime}$, put $\mu_{p}(X)=$ value at $p$ of the function $\sum_{n} \Phi_{\chi}\left(X \cap G_{n}\right)$. From $4.5(3), \mu_{p}$ is a finitely additive (non-negative) measure on $\mathcal{E}^{\prime}$, and $\mu_{p}\left(G_{n}\right) \leqq 1$. Further, if $X_{1}, X_{2}, \ldots$ is any sequence of disjoint sets in $\mathcal{E}^{\prime}$, and if $X=\bigcup X_{n} \in \mathcal{E}^{\prime}$, then $\mu_{p}(X)=\sum \mu_{p}\left(X_{n}\right)$ because $X$ is compact and each $X_{n}$ is open, so that all but a finite number of the sets $X_{n}$ must be empty. There is therefore $\left[1\right.$, p. 2] an extension of $\mu_{p}$ to a complete countably additive measure $\mu_{p}^{\prime}$ on a Borel field containing $B \mathcal{E}^{\prime}=\mathscr{B}^{\prime} .\left(^{7}\right)$ For $X \in \mathbb{B}^{\prime}$ define $\nu_{p}(X)$ $=\sum_{n} \mu_{p}^{\prime}\left(X \cap G_{n}\right)=\mu_{p}^{\prime}(X-Z)$. Then $\nu_{p}$ is a countably additive $\sigma$-finite measure on $\mathbb{B}^{\prime}$, which extends $\mu_{p}$. We assert:

(1) Given $X \in \mathcal{E}^{\prime}, \nu_{p}(X)=\left(\Phi_{\chi} X\right)(p)$ for each $p \in R-N_{X}$, where $N_{X} \in \Re^{\prime}$.

For say $X \in \mathcal{E}_{k}$; then $G_{n} \in \mathcal{E}_{1} \subset \mathcal{E}_{k}$ (we may assume $k>0$ ), so $X \cap G_{n} \in \mathcal{E}_{k}$ and $\nu_{p}(X)=\sum_{n} \mu_{p}\left(X \cap G_{n}\right)=\sum\left\{\right.$ value at $p$ of $\left.\Phi_{\chi}\left(X \cap G_{n}\right)\right\}$. Now each $\chi\left(X \cap G_{n}\right) \in \mathfrak{C}_{k}$, and also $\chi(X) \in \mathfrak{C}_{k}$ and $\sum \chi\left(X \cap G_{n}\right)=\chi X$ on $R-Z$ where $Z \in \Re_{k}$. Hence by $4.5(4)$ we have $\Phi\left(\sum \chi\left(X \cap G_{n}\right), 1 \leqq n \leqq m\right) \rightarrow \Phi \chi X$ on $R-N_{X}$ for some $N_{X} \in \mathfrak{N}_{k} \subset \mathfrak{N}^{\prime}$, and the assertion follows.

4.7 The outer measure function $\nu^{*}$. For arbitrary $X \subset R$ and $p \in R$, let $\nu_{p}^{*} X$ denote the outer measure of $X$ with respect to the measure $\nu_{p}$. We write $\nu^{*} X$ for the function on $R$ whose value at $p$ is $\nu_{p}^{*} X$. If $X \in \mathbb{B}^{\prime}, X$ is $\nu_{p}$-measurable for every $p \in R$. Generally, if $X$ is $\nu_{p}$-measurable except for a negligible set of $p^{\prime}$ s, we write $\nu X$ instead of $\nu^{*} X$.

( ) A "complete" measure is one for which all subsets of null sets are measurable. 
(1) If $X \in \mathfrak{r}^{\prime}, \nu X$ exists and is negligible.

We have $X=\cup X_{k}$ where $X_{k} \in \Re_{k}$, and we may suppose $k \geqq 1$. As the sets $G_{n}$ are disjoint and $\nu_{p}$-measurable for every $p$, we have $\nu^{*} X_{k}=\sum_{n} \nu^{*}\left(X_{k} \cap G_{n}\right)$, so it is enough to prove that $\nu^{*}\left(X_{k} \cap G_{n}\right)$ is $k$-negligible. By 3.2, $X_{k} \subset \cup_{m} \cap_{h} R x_{m h}$ where $x_{m h} \in E_{k}$ and, for fixed $m, x_{m 1} \geqq x_{m 2} \geqq \cdots$ and $\Lambda_{h} x_{m h}=0$. We have $G_{n}=R y_{n}$ where $y_{n} \in E_{1} \subset E_{k}$. Let $Z_{m n}=\bigcap_{h} R x_{m h} \cap G_{n}=\bigcap_{h} R\left(x_{m h} \wedge y_{n}\right)$. The sequence $\left\{\chi R\left(x_{m h} \wedge y_{n}\right)\right\}(h=1,2, \cdots)$ of functions of $\mathfrak{C}_{\mathbf{k}}^{+}$decreases monotonically, and its limit is 0 outside a $k$-negligible set; further, $\Phi_{\chi} R\left(x_{m 1} \wedge y_{n}\right)$ $\leqq \Phi \chi G_{n} \leqq 1$. Hence, by $4.5(4), \nu R\left(x_{m h} \wedge y_{n}\right)=\Phi \chi R\left(x_{m h} \wedge y_{n}\right) \rightarrow 0$ except on a $k$-negligible set as $h \rightarrow \infty$, proving $\nu^{*} Z_{m n}$ is $k$-negligible. As $X_{k} \cap G_{n} \subset \cup_{m} Z_{m n}$, this proves $\nu^{*}\left(X_{k} \cap G_{n}\right) k$-negligible, as required.

(2) If $X \in \Re^{\prime}, X$ is $\nu_{p}$-measurable for all $p \in R-N_{X}$, where $N_{X} \in \Re^{\prime}$; and $\nu X \in \mathcal{F}\left(\mathfrak{T}^{\prime}\right)$.

First suppose $X \in \mathcal{E}^{\prime}$. Then $X$ is $\nu_{p}$-measurable for all $p \in R$. Again, write $X_{n}=X \cap G_{n}$; we have $\nu X=\sum \nu X_{n}=\sum \Phi \chi X_{n}$ where $\Phi \chi X_{n} \in \mathfrak{C}^{\prime} \subset \mathcal{F}\left(\mathfrak{N}^{\prime}\right)$, from 3.3(3), showing that $\nu X \in \mathcal{F}\left(\mathfrak{N}^{\prime}\right)$.

Now suppose $X \in \mathbb{B}^{\prime}$. Again $X$ is $\nu_{p}$-measurable for all $p \in R$, and as above it is enough to prove that each $\nu\left(X \cap G_{n}\right) \in \mathcal{F}\left(\mathfrak{F}^{\prime}\right)$. Thus we may assume $X \subset G_{n}$. The $\mathfrak{T}^{\prime}$-measurability of $\nu X$ now follows by transfinite induction over the rank $\alpha$ of $X$ considered as in the Borel field generated by sets in $\mathcal{E}^{\prime}$ which are subsets of $G_{n}$; we use the facts that $X$ is a limit of a monotone sequence of sets of smaller rank and of finite measure, and that a (pointwise) limit of a sequence of functions in $\mathcal{F}\left(\mathfrak{M}^{\prime}\right)$ is in $\mathscr{F}\left(\mathfrak{N}^{\prime}\right)$.

Finally, if $X \in \mathfrak{M}^{\prime}, X=Y+Z^{\prime}$ where $Y \in \mathbb{B}^{\prime}, Z^{\prime} \in \mathfrak{N}^{\prime}$; if $p \in R-N_{X}$ where $N_{x}$ is negligible, $\nu_{p}^{*}\left(Z^{\prime}\right)=0$ by (1), and $X$ is $\nu_{p}$-measurable and $\nu_{p}(X)=\nu_{p}(Y)$. Thus $\nu(X)=\nu(Y) \in \mathcal{F}\left(\mathfrak{M}^{\prime}\right)$.

4.8 The operator $\Phi^{*}$. As a corollary to the last result, we have

(1) If $f \in \mathcal{F}\left(\mathfrak{T}^{\prime}\right)^{+}$, then $f$ is $\nu_{p}$-measurable except for a negligible set of $p$ 's.

We define $\Phi_{p}^{*} f=\inf \left\{\int_{R} g d \nu_{p} \mid g\right.$ is $\nu_{p}$-measurable and $\left.g \geqq f\right\}$. The function on $R$ whose value at $p$ is $\Phi_{p}^{*} f$ is denoted by $\Phi^{*} f$. It is easy to verify that, for arbitrary $X \subset R$,

$$
\Phi^{*} \chi X=\nu^{*} X .
$$

We deduce

(3) If $f \in \mathcal{F}\left(\mathfrak{T}^{\prime}\right)^{+}$, then $\Phi^{*} f \in \mathcal{F}\left(\mathfrak{M}^{\prime}\right)^{+}$.

For, by a familiar argument, $f=\sum \alpha_{n} \chi X_{n}$ where $\alpha_{n}>0, X_{n} \in \mathfrak{T I}^{\prime}$. By 4.7(2), $X_{n}$ is $\nu_{p}$-measurable for all $p \in R-N_{n}$ where $N_{n} \in \mathfrak{r}^{\prime}$. Put $N=\bigcup N_{n}$; then, if $p \in R-N, f$ is $\nu_{p}$-measurable and consequently $\Phi_{p}^{*} f=\int_{R} f d \nu_{p}$ $=\sum \alpha_{n} \nu_{p}\left(X_{n}\right)$ where, for each $n$, the function $\nu_{p}\left(X_{n}\right)$ of $p$ is in $\mathcal{F}\left(\mathfrak{T}^{\prime}\right)^{+}$by 4.7(2). Thus $\Phi^{*} f$ differs from an $\mathfrak{I}^{\prime}$-measurable function at most on $N$, and is therefore $\mathfrak{T}^{\prime}$-measurable.

(4) If $f \geqq 0$ and $[f] \in \mathfrak{r}^{\prime}$, then $\left[\Phi^{*} f\right] \in \mathfrak{r}^{\prime}$.

Let $[f]=X$. By 4.7(1), $\nu_{p}^{*} X=0$ for all $p \in R-N$ where $N \in \mathfrak{I}^{\prime}$. If $p \in R-N$, 
we may take $g=\infty \chi X$ in the definition of $\Phi_{p}^{*} f$, showing $\Phi_{p}^{*} f=0$ for $p \in R-N$.

(5) If $f_{n} \in \mathcal{F}\left(\mathscr{T N}^{\prime}\right)^{+}(n=1,2, \cdots)$, then $\Phi_{p}^{*}\left(\sum f_{n}\right)=\sum \Phi_{p}^{*} f_{n}$ except for a negligible set of $p$ 's.

The proof resembles that of (3). We immediately deduce:

(6) If $f_{n} \in \mathscr{F}\left(\mathfrak{T}^{\prime}\right)+(n=1,2, \cdots), f_{1} \geqq f_{2} \geqq \cdots$, and $\Phi_{p}^{*} f_{1}<\infty$ except on a negligible set, then $\Phi_{p}^{*}\left(\lim f_{n}\right)=\lim \Phi_{p}^{*} f_{n}$ except on a negligible set.

If $f \in \mathrm{e}^{\prime+}$, the value of $\Phi f$ at $p \in R$ is denoted by $\Phi_{p} f$; similarly we define $\Phi_{p}^{m} f$.

(7) If $f \in \mathfrak{C}^{\prime+}$, then $\Phi_{p}^{*} f=\Phi_{p} f$ if $p \in R-N$ where $N \in \mathfrak{T}^{\prime}$.

Say $f \in \mathcal{C}_{k}$. By 3.4(5) we may write $f=\sum \sigma_{n} \chi X_{n}+g$ where $\sigma_{n}>0, X_{n} \in \mathcal{E}_{k}$, $g \geqq 0$ and $[g] \in \Re_{k} \subset \mathfrak{r}^{\prime}$. From 4.5(4), if $p \in R-N_{1}$ where $N_{1} \in \mathfrak{N}_{k}$, $\Phi_{p} f=\sum \Phi_{p}\left(\sigma_{n} \chi X_{n}\right)=\sum \sigma_{n} \nu_{p}\left(X_{n}\right)$ by 4.6 , if $p \notin N_{2}$, where $N_{2} \in \Re_{k}$. On the other hand, from (4), $\Phi_{p}^{*} g=0$ except on $N_{3} \in \mathfrak{r}^{\prime}$, and outside $N_{3}$ we have that $f$ is $\nu_{p}$-measurable and consequently $\Phi_{p}^{*} f=\sum \sigma_{n} \int \chi X_{n} d \nu_{p}=\sum \sigma_{n} \nu_{p}\left(X_{n}\right)$. Thus $\Phi_{p}^{*} f=\Phi_{p} f$ if $p \in R-\left(N_{1} \cup N_{2} \cup N_{3}\right)$.

Since $\Phi^{*}$ maps $\mathscr{F}\left(\mathscr{T}^{\prime}\right)^{+}$in itself, the iterates $\Phi^{*_{m}}(m=1,2, \cdots)$ are all defined; it is easy to see that properties (3)-(6) apply to $\Phi^{* m}$, and similarly (7) gives (with a little more trouble):

$\left(7^{\prime}\right)$ If $f \in \mathfrak{C}^{\prime+}, \Phi^{* m} f$ and $\Phi^{m} f$ differ only on a negligible set.

4.9 Support properties of $\Phi^{*}$. We list the following properties of $\Phi^{*}$ for later use; they all follow easily from the foregoing. Throughout, it is assumed that $f, f_{1}, f_{2}, \cdots \in \mathscr{F}\left(\mathscr{M}^{\prime}\right)^{+}$.

(1) $\left[\Phi^{* m_{f}}\right] \in \mathfrak{N}^{\prime}$.

(2) If $[f] \in \mathfrak{T}^{\prime},\left[\Phi^{* m_{f}}\right] \in \mathfrak{T}^{\prime}$.

(3) $\cup_{n}\left[\Phi^{*_{m}} f_{n}\right] \subset\left[\Phi^{*_{m}} \sup f_{n}\right] \subset\left[\Phi^{*_{m}} \sum f_{n}\right]$, and

$$
\left[\Phi^{* m} \sum f_{n}\right]-\cup_{n}\left[\Phi^{* m} f_{n}\right] \in \Re^{\prime} .
$$

(4) If $\left[f_{1}\right]+\left[f_{2}\right] \in \mathfrak{r}^{\prime}$, then $\left[\Phi^{* m_{1}} f_{1}\right]+\left[\Phi^{* m_{2}} f_{2}\right] \in \mathfrak{r}^{\prime}$.

In particular,

$$
\left[\Phi^{* m} f\right]+\left[\Phi^{* m} \chi[f]\right] \in \Re^{\prime} .
$$

4.10 The set-operator $I$. As a preliminary to defining the final ideal $\mathfrak{N}$ of "null sets," we define $I(X)$, for $X \in \mathfrak{N}^{\prime}$, by: $I(X)=\left[\sum \Phi^{* m} \chi X\right]$, where $m=0,1,2, \cdots$. Taking $m=0$ shows

$$
I(X) \supset X \text {. }
$$

The following results follow easily with the aid of 4.9. It is assumed throughout that $X, X_{1}, X_{2}, \cdots \in \mathfrak{M}^{\prime}$.

(2) $I(X) \in \mathfrak{T}^{\prime}$.

(3) If $X \in \mathfrak{r}^{\prime}, I(X) \in \mathfrak{r}^{\prime}$.

(4) $I(X)=U\left[\Phi^{* m} \chi X\right](m \geqq 0)$.

(5) If $X \subset Y, I(X) \subset I(Y)$.

(6) $I\left(\cup X_{n}\right)=\cup I\left(X_{n}\right) \cup N$, where $N \in \mathfrak{r}^{\prime}$. 
(7) $I(X+Y) \supset I(X)+I(Y)$ mod $\Re^{\prime}$; hence if $X+Y \in \mathfrak{r}^{\prime}$,

$$
I(X)+I(Y) \in \Re^{\prime} .
$$

(8) $I(I(X))=I(X) \bmod \Re^{\prime}$.

(9) If $f \in \mathscr{F}\left(\mathfrak{T}^{\prime}\right)^{+}$, then $\left[\sum \Phi^{*_{m}} f\right]=I[f] \bmod \Re^{\prime}$, and hence

$$
I\left[\Phi^{*} f\right] \subset I[f] \bmod \Re^{\prime} .
$$

As the last four of these statements are less trivial than the others, we sketch their proofs.

Proof of (6). By (4) and 4.9(3),

$$
I\left(\bigcup X_{n}\right)=\bigcup_{m}\left[\Phi^{*_{m}} \chi \cup X_{n}\right] \subset \underset{m}{\bigcup}\left[\Phi^{*_{m}} \sum \chi X_{n}\right]=\bigcup_{m}\left\{\bigcup_{n}\left[\Phi^{*{ }_{m}} \chi X_{n}\right] \cup Z_{m}\right\}
$$

where $Z_{m} \in \mathfrak{T}^{\prime},=\mathrm{U}_{m, n}\left[\Phi^{* m} \chi X_{n}\right] \cup Z^{\prime}=\cup I\left(X_{n}\right) \cup Z^{\prime}$ where $Z^{\prime} \in \mathfrak{T}^{\prime}$. But $I\left(\cup X_{n}\right)$ $\supset I\left(X_{n}\right)$, by (5).

Proof of (7). By (6), $I(X)=I(X \cap Y) \cup I(X-Y) \cup N_{1}, I(Y)=I(X \cap Y)$ $\cup I(Y-X) \cup N_{2}$, where $N_{1}, N_{2} \in \mathfrak{x}^{\prime} ;$ so $I(X)+I(Y) \subset I(X-Y) \cup I(Y-X)$ $\cup N_{1} \cup N_{2} \subset I(X+Y) \cup\left(N_{1} \cup N_{2}\right)$ by (5).

Proof of (8). Using (4), (6) and 4.9(5), we find

$$
I(I(X))=\bigcup_{m} \bigcup_{n}\left[\Phi^{*_{n}} \chi\left[\Phi^{*_{m}} \chi(X)\right]\right]=\bigcup_{m, n}\left[\Phi^{*_{m+n}} \chi X\right] \bmod \mathfrak{\Upsilon}^{\prime}=I(X) \bmod \mathfrak{\Upsilon}^{\prime} .
$$

Proof of (9). From (4) and 4.9(5), $I[f]=\left[\Phi^{* m} \chi[f]\right]=U\left[\Phi^{* m} f\right] \bmod \Re^{\prime}$ $=\left[\sum \Phi^{* m} f\right]$. Hence $\left[\Phi^{*} f\right]=I[f]$ mod $\Re^{\prime}$, giving (from (3) and (8)) $I\left[\Phi^{*} f\right]$ $C I(I[f]) \bmod \Re^{\prime}=I[f] \bmod \mathfrak{\varkappa}^{\prime}$.

5. Proof of Theorem 1 concluded.

5.1 The $\sigma$-ideal $\Re$. Define $\Re=\left\{X \mid\right.$ there exists $Y \in \mathfrak{N}^{\prime}$ such that $X \subset Y$ and $I(Y) \in \mathcal{K} R\}$. We have at once:

(1) $\Re$ is a $\sigma$-ideal. (From $4.10(6)$.)

(2) $\Re^{\prime} \subset \Re \subset \Re R$. (From 4.10(3) and 4.10(1).)

(3) If $X \in \mathcal{E}^{\prime} \cap \Re, X$ is empty. (From (2).)

(4) If $f \in \mathcal{F}\left(\mathfrak{T}^{\prime}\right)+$ and $[f] \in \Re$, then $\left[\Phi^{*} f\right] \in \Re$. (From 4.10.)

(5) If $f, g \in \mathscr{F}\left(\mathfrak{N}^{\prime}\right)^{+}$and $[f]+[g] \in \Re$, then $\left[\Phi^{*} f\right]+\left[\Phi^{*} g\right] \in \Re$.

For let $R-X=[f] \cap[g]$, and put $f=f \chi X+f^{\prime}, g=g \chi X+g^{\prime}$. Then $\left[f^{\prime}\right]$ $=\left[g^{\prime}\right]$, so $\left[\Phi^{*} f^{\prime}\right]=\left[\Phi^{*} g^{\prime}\right] \bmod \mathfrak{T}^{\prime}$, by $4.9(4)$. Also $\Phi^{*} f=\Phi^{*} f^{\prime}+\Phi^{*} f \chi X \bmod \mathfrak{T}^{\prime}$ $=\Phi^{*} f^{\prime} \bmod \Re$ by (4) and (2). Thus, modulo $\Re,\left[\Phi^{*} f\right]=\left[\Phi^{*} f^{\prime}\right]=\left[\Phi^{*} g^{\prime}\right]=\left[\Phi^{*} g\right]$.

5.2 The algebra $E$. Now put $B=\mathfrak{T}^{\prime}+\mathfrak{N}$; this is a Borel field containing $\Re$. Define $E=\leftrightarrow / \Re$, a Boolean $\sigma$-algebra. Since $\mathfrak{T}^{\prime}=\mathbb{B}^{\prime}+\mathfrak{r}^{\prime}$ and $\Re \supset \Re^{\prime}$, we have $\mathbb{B}=\mathbb{B}^{\prime}+\Re$, and a typical element of $E$ is thus the class of sets $(X)+\Re$ $(=\{X+N \mid N \in \mathfrak{\Re}\})$ where $X \in \mathbb{B}^{\prime}=\mathbb{B} R$. We now prove

(1) E satisfies the countable chain condition.

Suppose $a$ is an uncountable family of sets $A \in \mathbb{B}^{\prime}$, none of which is in $\Re$, 
but such that whenever $A_{1}, A_{2}$ are distinct members of $Q$ then $A_{1} \cap A_{2} \in \Re$; we must derive a contradiction. We may suppose that $a$ consists of just $\boldsymbol{\aleph}_{1}$ sets; well-order $\alpha$ as $\left\{A_{\alpha} \mid \alpha<\omega_{1}\right\}$, and let $A_{\alpha}^{\prime}=A_{\alpha}-\cup\left\{A_{\beta} \mid \beta<\alpha\right\}$; then $A_{\alpha}^{\prime} \in \mathbb{B}^{\prime}, A_{\alpha}-A_{\alpha}^{\prime} \in \mathcal{N}$, and distinct sets $A_{\alpha}^{\prime}$ are disjoint. Thus, replacing $a$ by $\left\{A_{\alpha}^{\prime} \mid \alpha<\omega_{1}\right\}$, we may further assume that $\alpha$ consists of disjoint sets.

For each $A \in Q$, there is a least $n \geqq 0$ such that $\left[\Phi^{*_{n}} \chi A\right]$ is of second category in $R$ (else $I(A) \in \mathcal{K} R$ and $A \in \mathfrak{N}$ ). Let $a_{k}$ be the subfamily of $a$ for which this $n$ has the value $k$; then $Q_{k}$ must be uncountable for some $k$. If $k=0$, we have that each $A \in a_{0}$ is itself of second category; but (2.1(2)) each $A \in \mathbb{B}^{\prime}$ has the form $R a+H$ where $a \in E^{\prime}$ and $H \in \mathcal{K} R$, and if $A$ is of second category then $a \neq 0$. So if $a_{0}$ is uncountable, $E^{\prime}$ would not satisfy the countable chain condition. We may therefore assume that $a_{k}$ is uncountable for some $k \geqq 1$.

By 4.5(7) and 4.8(7) there exist sets $Y_{1}, Y_{2}, \cdots \in \varepsilon_{k}$ such that $R-U Y_{n}$ $\in \Re_{k} \subset \Re^{\prime}$ and $\Phi^{* k} \chi Y_{n} \leqq 1 \bmod \Re^{\prime}$. For every $A \in \mathbb{B}^{\prime}$ we have $\left[\Phi^{* k} \chi A\right]$ $=\mathrm{U}_{n}\left[\Phi^{* k} \chi\left(A \cap Y_{n}\right)\right] \bmod \mathfrak{T}^{\prime}$, by $4.9(2)$ and 4.9(3); hence if $A \in \mathrm{Q}_{k}$ we have that $\left[\Phi^{* k} \chi\left(A \cap Y_{n}\right)\right]$ is of second category for some $n$. There is therefore some $n$, which we may assume to be 1 , to which uncountably many sets $A \in a_{k}$ correspond; we replace each such $A$ by $A \cap Y_{1}$, and thus obtain an uncountable family $Q^{\prime} \subset \mathbb{B}^{\prime}$ of disjoint sets satisfying:

(2) If $A \in Q^{\prime}$, then $A \subset Y_{1}$ and [ $\left.\Phi^{* k} \chi A\right]$ is of second category.

From 4.9(1), there exists for each $A \in Q^{\prime}$ a positive integer $n(A)$ and a set $W(A) \in \mathfrak{T}^{\prime}$ of second category such that $\Phi^{* k} \chi A \geqq(1 / n(A)) \chi W(A)$. There is a positive integer $h$ such that $n(A)=h$ for uncountably many sets in $Q^{\prime}$; we may thus assume further that

(3) If $A \in a^{\prime}, \Phi^{* k} \chi A \geqq(1 / h) \chi W(A)$.

For each subset $\mathscr{X} \subset \boldsymbol{Q}^{\prime}$, put $W(\mathfrak{X})=\cap\{W(A) \mid A \in \mathfrak{X}\}$. Then:

(4) If $x$ has more than $h$ elements, $W(x)$ is of first category.

It is enough to prove this when $x$ has $h+1$ elements $A_{0}, A_{1}, \cdots, A_{h}$. As these sets are disjoint, we have, modulo $\Re$-negligible functions, from (3), $(h+1) \chi W(x) \leqq \sum\left\{h \Phi^{* k} \chi A_{i} \mid 0 \leqq i \leqq h\right\}=h \Phi^{* k}\left(\sum \chi A_{i}\right)$ (see end of 4.8) $\leqq h \Phi^{* k} \chi\left(\cup A_{i}\right) \leqq h \Phi^{* k} \chi Y_{1} \leqq h$, proving $W(x) \in \Re \subset \varkappa R$.

Consider now the family $\{y\}$ of maximal subsets $y$ of $a^{\prime}$ for which $W(\mathcal{Y})$ is of second category; each $A \in \alpha^{\prime}$ is in at least one $\mathcal{Y}$ (from (4), since $A$ is itself of second category), and each $Y$ contains at most $h$ sets $A \in Q^{\prime}$. Further, if $\mathfrak{Y}_{1} \neq \mathfrak{Y}_{2}, W\left(\mathcal{Y}_{1}\right)$ and $W\left(\mathcal{Y}_{2}\right)$ are in $\mathfrak{T}^{\prime}$, are both of second category, and have intersection of first category. By an argument similar to that used above for $k=0$, there are only countably many sets $W(Y)$, and therefore only countably many sets $\mathcal{Y}$, each with $\leqq h$ elements. Thus $\alpha^{\prime}=U \mathcal{Y}$ is countable, giving the desired contradiction.

5.3 The operator $\phi$. Let $F=F(E)$; we define a mapping $\phi$ of $F^{+}$in $F^{+}$which will be proved to satisfy the requirements of Theorem 1 (4.1). The elements of $F$ are of the form $\tilde{f}=f+Z(\mathscr{X})$, where $f \in \mathcal{F}\left(\mathfrak{T}^{\prime}\right)$; here $f+Z(\mathscr{X})$ denotes the 
family of all functions $f+h$ where $h$ is $\Re$-negligible. Since $B=B^{\prime}+\mathscr{N}$, we may require $f \in \mathcal{F}\left(B^{\prime}\right)$ here.

Define $\phi \tilde{f}=\Phi^{*} f+Z(\Re)$, where $\tilde{f}=f+Z(\Re)$. From $4.8(3)-(5)$, this definition is single-valued and $\phi$ maps $F^{+}$in itself. Further, $\phi$ is countably additive from 4.8(5), and $\sigma$-finite from 4.5(7) and 4.8(7); $\phi$ is thus an $F$-integral on $F$ (2.6). To verify that $\phi$ is a cylinder extension of $\phi_{0}$, we first observe that the correspondence $x \leftrightarrow(R x)+\mathscr{N}$ is (from 5.1(3)) a finitely additive isomorphism between $E^{\prime}$ and a finitely additive subalgebra of $E$ which, restricted to $E_{k}$, is a complete isomorphism (because $\Re_{\supset} \supset \mathscr{N}^{\prime} \supset \mathscr{N}_{k}$ ). We may identify $E_{k}$ with the complete subalgebra $\left\{R x+\Re \mid x \in E_{k}\right\}$ of $E$ (equivalently, we take $\left.E_{k}=\left(B_{k}+\mathscr{T}\right) / \mathscr{T}\right)$; and similarly we may identify $F_{k}$ with the set of function classes $f+\mathcal{Z}(\mathfrak{X}), f \in \mathcal{F}\left(\mathbb{B}_{k}\right)$ - that is, we realise $F_{k}$ as $\mathscr{F}\left(R, B_{k}+\mathscr{N}, \mathfrak{X}\right)$. The cylinder mapping of $F_{k}$ in $F$ is now the identity mapping of $F_{k}$. If $f \in \mathcal{F}\left(\mathbb{B}_{k}\right)^{+}$, so that $\tilde{f}=f+Z(\mathfrak{Z})$ is a typical element of $F_{k}^{+}$, we again have that $R_{k} \tilde{f}$ is the unique $k$-continuous function in $\tilde{f}$ (compare 4.4)( $\left.{ }^{8}\right)$. Now if $f \in \mathcal{F}\left(\Theta_{k}\right)^{+}$, $\Phi^{*} f=\Phi^{*} R_{k} \tilde{f} \bmod \mathfrak{H}$ by $4.8(4),(5),=\Phi R_{k} \tilde{f} \bmod \mathfrak{T}$ by $4.8(7)=R_{k} \phi_{k} f \bmod \mathfrak{N}$ by definition of $\Phi$, so that $\phi_{k} \tilde{f}=\left(\Phi^{*} f\right)^{\sim}=\phi f$. That is, $\phi$ is a cylinder extension of $\phi_{k}(k=0,1,2, \cdots)$, and in particular of $\phi_{0}$.

5.4 Full homogeneity. All that remains is to show that $\phi$ is fully homogeneous. Write $\lambda x=\phi \chi x$ for $x \in E$; thus $\lambda$ is countably additive and $\sigma$-finite. Let $z_{0}=\bigvee\{z \mid z \in E, \lambda z=0\}, z_{1}=e-z_{0}, E^{1}=\left\{z \mid z \in E, z \leqq z_{1}\right\}$. We first show:

(1) Given $x \in E$, there exists $\sigma x \in E^{1}$ such that, for all $y \in E$,

$$
\lambda(y \wedge \sigma x)=(\lambda y) \chi x .
$$

(The element $\sigma x$ is in fact unique, but we do not need this.)

For let $H$ be the set of elements $x \in E$ for which such a $\sigma x$ exists. Then

(2) if $x \in H$ and $y \in E, \lambda\left\{y \wedge\left(z_{1}-\sigma x\right)\right\}=(\lambda y) \chi(-x)$.

For suppose first that $\lambda y \ll \infty$. Then

$$
\begin{aligned}
\lambda(y)=\lambda\left(y \wedge z_{1}\right) & =\lambda(y \wedge \sigma x)+\lambda\left(y \wedge\left(z_{1}-\sigma x\right)\right) \\
& =(\lambda y) \chi x+\lambda\left(y \wedge\left(z_{1}-\sigma x\right)\right),
\end{aligned}
$$

so $\lambda\left(y \wedge\left(z_{1}-\sigma x\right)\right)=(\lambda \dot{y})(1-\chi x)=(\lambda y) \chi(-x)$.

In the general case, we know $y=\bigvee_{y_{n}}(n=1,2, \cdots)$ where $\lambda y_{n} \ll \infty$, and the elements $y_{n}$ can be assumed disjoint. Then $\lambda\left(y_{n} \wedge\left(z_{1}-\sigma x\right)\right)=\left(\lambda y_{n}\right) \chi(-x)$, and summation gives (2).

This shows that if $x \in H$ then $-x \in H$, with $\sigma(-x)=z_{1}-\sigma x$.

Next let $x_{n} \in H(n=1,2, \cdots), y \in E$, and suppose $\lambda(y) \ll \infty$. Then $\lambda\left(y \wedge \wedge \sigma x_{n}\right) \leqq \lambda\left(y \wedge \sigma x_{n}\right)=(\lambda y) \chi x_{n}$ for all $n$, and therefore

$$
\lambda\left(y \wedge \wedge \sigma x_{n}\right) \leqq(\lambda y) \chi\left(\Lambda x_{n}\right) .
$$

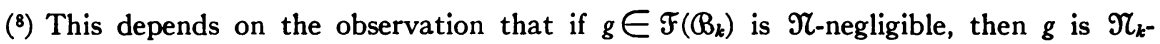
negligible. For $g=h \bmod \mathfrak{T}_{k}$ where $h$ is $k$-continuous; being continuous and $\mathcal{K} R$-negligible, $h$ must be 0 . 
But $\lambda\left\{y \wedge\left(z_{1}-\wedge \sigma x_{n}\right)\right\}=\lambda\left\{y \wedge \vee\left(z_{1}-\sigma x_{n}\right)\right\} \leqq \sum \lambda\left\{y \wedge\left(z_{1}-\sigma x_{n}\right)\right\}$ $=\sum(\lambda y) \chi\left(-x_{n}\right)$ by $(2)=\lambda y \sum \chi\left(-x_{n}\right)$, and thus $\lambda\left\{y \wedge\left(z_{1}-\Lambda \sigma x_{n}\right)\right\}$ $\leqq \inf \left\{\lambda y, \lambda y \sum \chi\left(-x_{n}\right)\right\}$; that is,

$$
\lambda\left(y \wedge\left(z_{1}-\Lambda \sigma x_{n}\right)\right) \leqq(\lambda y) \chi \vee\left(-x_{n}\right) .
$$

But $\lambda\left\{y \wedge \wedge \sigma x_{n}\right\}+\lambda\left\{y \wedge\left(z_{1}-\Lambda \sigma x_{n}\right)\right\}=\lambda\left(y \wedge z_{1}\right)=\lambda y \ll \infty$. Adding (3) and (4), we see that both inequalities must be equalities; in particular (3) becomes

$$
\lambda\left(y \wedge \bigwedge \sigma x_{n}\right)=(\lambda y) \chi\left(\bigwedge x_{n}\right)
$$

if $\lambda y \ll \infty$.

The restriction $\lambda y \ll \infty$ is easily removed, as before, so (5) shows that $\bigwedge x_{n} \in H$, with $\sigma\left(\Lambda x_{n}\right)=\Lambda \sigma x_{n}$.

Thus $H$ is a $\sigma$-subalgebra of $E$. Further,

$$
H \text { contains each } E_{k}(k=0,1,2, \cdots) \text {. }
$$

For (4.2(3)) $c_{k+1, k} \phi_{k+1}$ is a fully homogeneous $F_{k}$-integral on $F_{k+1}$; we apply [4, p. 175, Lemma 4] to its strict form, taking $\sigma x=\pi\left(z_{1}, x\right)$ for $x \in E_{k+1}$, and obtain $c_{k+1, k} \phi_{k+1} \chi_{k+1}(y \wedge \sigma x)=\left(c_{k+1, k} \phi_{k+1} \chi_{k+1} y\right) \chi_{k+1} x, \chi_{k+1}$ denoting the characteristic function in $F_{k+1}$. We use the same realisations of $F_{k}, F_{k+1}$ as at the end of 5.3; the cylinder mappings become identities, $\chi_{k+1} y=\chi y$ for $y \in E_{k+1}$, and because $\phi$ is a cylinder extension of $\phi_{k+1}$ it follows that $\phi \chi(y \wedge \sigma x)$ $=(\phi \chi y) \chi x$ for $x \in E_{k+1}$; thus $E_{k} \subset E_{k+1} \subset H$.

Now let $\mathfrak{H}=$ family of all sets $X \in \mathbb{B}^{\prime}$ such that $(X)+\mathfrak{x} \in H$. Then $\mathfrak{H}$ is a Borel field containing $\cup \mathcal{E}_{k}=\mathcal{E}^{\prime}$, so $\mathfrak{x} \supset \mathbb{B}^{\prime}$. That is, $(X)+\Re \in H$ for all $X \in \mathbb{B}^{\prime}$, proving $E \subset H$. This establishes (1).

Now, given $x \in E$ and $g \in F^{+}$such that $g \leqq \lambda x$, we must find $y \leqq x$ in $E$ such that $\lambda y=g$. We may of course assume $g>0$; and it is enough to show that there then exists a nonzero $z \leqq x$ in $E$ such that $\lambda z \leqq g$, as then an "exhaustion" argument, based on the countable chain condition (cf. [2, p. 283]), produces the required element $y$. We may further suppose that $x \leqq a_{1}$ where $a_{1} \in E_{1}$ and $\lambda a_{1} \leqq 1$. For $c_{10} \phi_{1}=\phi_{1}$ is fully homogeneous on $F_{1}$ and $\phi$ extends $\phi_{1}$; hence (as in the proof of 4.6(7)) disjoint elements $a_{1}, a_{2}, \ldots \in E_{1}$ exist such that $V a_{n}=e$ and $\lambda a_{n} \leqq 1$. Since $\sum \lambda\left(x \wedge a_{n}\right) \geqq g>0$, there exists $n$ such that $\left[\lambda\left(x \wedge a_{n}\right)\right] \wedge[g] \neq 0$; we may suppose $n=1$, and then have $g_{1}=\lambda\left(x \wedge a_{1}\right) \wedge g$ $>0$; we replace $x$ by $x \wedge a_{1}$ and $g$ by $g_{1}$. For some positive integer $m$ we have $g \geqq(1 / m) \chi w$ for some nonzero $w \in E$. Because of the full homogeneity of $\phi_{1}$, there exist disjoint elements $b_{1}, b_{2}, \cdots, b_{m} \in E_{1}$ such that $V b_{i}=a_{1}$ and $\lambda b_{i}$ $=(1 / m) \lambda a_{1}^{a}$. Since $x \leqq \bigvee b_{i}, \quad \sum \lambda\left(b_{i} \wedge x \wedge \sigma w\right)=\sum \lambda\left(b_{i} \wedge x\right) \chi w=(\lambda x) \chi w \geqq g \chi w$ $\geqq(1 / m) \chi w>0$. Hence, for some $i, 0<\lambda\left(b_{i} \wedge x \wedge \sigma w\right) \leqq \lambda\left(b_{i} \wedge \sigma w\right)=\left(\lambda b_{i}\right) \chi w$ $\leqq(1 / m) \chi w \leqq g$, and we take $z=b_{i} \wedge x \wedge \sigma w$. This completes the proof of Theorem 1.

\section{Extensions of measures on measure algebras.}

THEOREM 2. Let $A$ be a $(\sigma-)$ subalgebra of a measure algebra $(E, \mu) .\left(^{\circ}\right)$ Let $\lambda$

( ${ }^{\vartheta}$ By saying that $(E, \mu)$ is a measure algebra, we imply that $\mu$ is $\sigma$-finite and positive on $E$. 
be a $\sigma$-finite positive measure on $A$. Then there exists a $\sigma$-finite positive measure $\lambda^{*}$ on $E$ which extends $\lambda$.

In what follows, it is understood that all measures are to be complete and $\sigma$-finite, and that the sets and functions used are measurable.

By [3, Theorem $2 \mathrm{~b}, \mathrm{p} .149],(E, \mu)$ has a realisation of the following form. We can realise $A$ algebraically as the measure algebra of a measure space $(S, \nu)$ (the measure $\nu$ has no simple relation to $\mu$ ), and can find a measure space $(T, m)$ and a subset $K$ of the product space $S \times T$ (to which we give the usual product measure), in such a way that there is a measure-preserving isomorphism $\theta$ of $(E, \mu)$ onto a certain Borel field of subsets of $K$ modulo null sets, and further if $a \in A$ then $\theta a$ is the class of the "cylinder set" $(U \times T) \cap K$, where $U$ is any subset of $S$ in the class $a$.

By the Radon-Nikodym theorem, there exists a non-negative function $f$ on $S$ such that, for each $U \subset S, \lambda(U)=\int_{U} f(s) d \nu(s)$. (Here $\lambda(U)$ means $\lambda a$ where $a$ is the class of $U$ modulo null sets.) Write $T=U T_{n}(n=1,2, \cdots)$, where the sets $T_{n}$ are disjoint and $m\left(T_{n}\right)$ is positive and finite. Define

$$
P(s)=\int_{T} \chi_{K}(s, t) \sum\left\{\left(\chi T_{n}\right) / 2^{n} m\left(T_{n}\right)\right\} d m(t) ;
$$

this is defined and $\leqq 1$ for almost all $s \in S$. Further, $P(s)>0$ almost everywhere, since if $P(s)=0$ for all $s \in U$, the set $(U \times T) \cap K$ is null, showing that $U$ is in the class of $o \in A$-that is, $\nu(U)=0$. Now define, for $X \subset S \times T$,

$$
\lambda^{*}(X)=\iint_{S \times T}(f(s) / P(s))(\chi X) \sum\left(\chi T_{n} / 2^{n} m\left(T_{n}\right)\right) d \nu(s) d m(t) .
$$

Then, applying $\theta$, we see that $\lambda^{*}$ gives a $\sigma$-finite positive measure on $E$. To show that $\lambda^{*}$ extends $\lambda$ on $A$, we verify (by a straightforward calculation) that if $U \subset S, \lambda^{*}((U \times T) \cap K)=\lambda U$.

7. Extensions of operators for measure algebras. In this section we prove that if we start with a measure algebra in Theorem 1, then we can arrange to end up with a measure algebra. More precisely:

TheOREM 3. If, in Theorem $1, E_{0}$ is a measure algebra $\left({ }^{9}\right)$ with measure $m_{0}$, the algebra $E$ can be chosen so that it is also a measure algebra, with measure $m$ extending $m_{0}$.

Proof. Since $m_{0}$ is $\sigma$-finite on $E_{0}$, we can find an equivalent finite measure $m_{0}^{\prime}$ on $E_{0}$; say $m_{0}^{\prime}(e)=1$. We use the entire argument of $\S 4$ (but not of $\S 5$ ), with the following additions. We first observe that $E_{1}$ can be taken to be a measure algebra, say with measure $m_{1}$. For let $z_{0}=\bigvee\left\{x \mid x \in E_{0}, \phi_{0} \chi x=0\right\}$, $z_{1}=e-z_{0}$. The "strict form" $\phi_{0 s}$ of $\phi_{0}$ is defined on the function space on the principal ideal $E_{0}\left(z_{1}\right)=\left\{x \mid x \in E_{0}, x \leqq z_{1}\right\}$, and its range is the function space on the principal ideal $E_{0}(\phi 1]$. The construction of $E_{1}$ depended in the first 


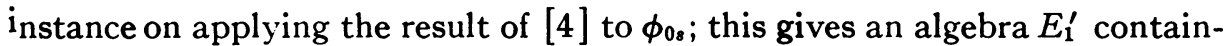
ing $E_{0}\left(z_{1}\right)$ as a subalgebra, and a fully homogeneous strict extension $\phi_{0}^{*}$ of $\phi_{0 s}$ to an operator from $F\left(E_{1}^{\prime}\right)$ to $F\left(E_{0}[\phi 1]\right)$. We then take $E_{1}=$ direct sum of $E_{1}^{\prime}$ and $E_{0}\left(z_{0}\right)$, imbedding $E_{0}$ in $E_{1}$ in the obvious way; $\phi_{1}$ is defined by $\phi_{1} f$ $=c_{01} \phi_{0 s}\left(f \chi z_{1}\right)$. By [4, Theorem 5], $E_{1}^{\prime}$ is isomorphic to a principal ideal in a product $J \times E_{0}[\phi 1]$, where $J$ is a numerical measure algebra. By $[3 ; 2 ; 4]$, if we give $E_{0}[\phi 1]$ the measure $m_{0}^{\prime}$, then $J \times E_{0}[\phi 1]$ with the usual product measure induces a (positive, $\sigma$-finite) measure (say) $m_{1}$ on $E_{1}^{\prime}$. We extend $m_{1}$ to $E_{1}$ by using $m_{0}^{\prime}$ on $E_{0}\left(z_{0}\right)$. By Theorem 2, there is a (positive) measure $m_{1}^{\prime}$ on $E_{1}$ which extends $m_{0}^{\prime}$. Note that $m_{1}^{\prime} \leqq 1$ on $E_{1}$, because $m_{1}^{\prime}(e)=m_{0}^{\prime}(e)=1$.

In this way, we may suppose that all the algebras $E_{k}$ of 4.2 are measure algebras, the measure $m_{k+1}^{\prime}$ on $E_{k+1}$ extending $m_{k}^{\prime}$ on $E_{k}$. Their common value gives a finitely additive measure $m^{\prime}$ on $E^{\prime}$, and hence on the family $\mathcal{E}^{\prime}$ of sets $R x, x \in E^{\prime}$, in the representation space $R$ of $E^{\prime}$ (cf. 4.3). By the same reasoning as in 4.6, we extend $m^{\prime}$ to a complete, countably additive measure (still denoted by $m^{\prime}$ ) on a Borel field containing $\mathscr{B}^{\prime}$; note that $m^{\prime}(R)=1$. Let $\Re^{\circ}$ denote the family of subsets of $R$ with zero $m^{\prime}$-measure. We show:

$$
\Re^{0} \supset \Re_{k}, \quad(k=0,1,2, \cdots) .
$$

For, by 2.3(4), each $X \in \Re_{k}$ is contained in a set of the form $\xi^{-1} Y$, where $Y \in \Re S \cap \leftrightarrow S$. By 3.2, $Y \subset \cup_{m} \cap_{n} S x_{m n}$ where $x_{m n} \in E_{k}, x_{m 1} \geqq x_{m 2} \geqq \cdots$ and $\bigwedge_{n} x_{m n}=0$. Thus $X \subset \xi^{-1} Y \subset \bigcup_{m} \bigcap_{n} R x_{m n}$ by 2.2(1); now, as $m_{k}^{\prime}$ is finite, $m_{k}^{\prime} x_{m n} \rightarrow 0$ as $n \rightarrow \infty$, so $m^{\prime} \bigcap_{n} R x_{m n}=0$ for each $m$, proving $m^{\prime} X=0$ if $X \in \mathscr{N}_{k}$.

It follows at once that

$$
\mathfrak{N}^{0} \supset \mathfrak{N}^{\prime} .
$$

Define $\mathscr{N}=\left\{X \mid X \subset Y\right.$ for some $Y \in \mathfrak{N}^{\prime}$ such that $\left.I(Y) \in \mathfrak{N}^{0}\right\}$. It is easily verified that all the properties in 5.1 continue to apply for this modified definition of $\Re$, except that in 5.1(2) we no longer have $\mathscr{K} \subset \mathcal{K} R$. But instead we have

$$
\mathfrak{X} \subset \mathfrak{N}^{0},
$$

because if $X \in \mathfrak{N}$ then $X \subset Y \subset I(Y)$ where $I(Y) \in \Re^{0}$. Hence 5.1(3) continues to hold.

For each $f \in F\left(\mathscr{T T}^{\prime}\right)^{+}$, put

$$
\psi f=\sum_{1}^{\infty} 2^{-n} \Phi^{*}\left(f \chi G_{n}\right),
$$

the sets $G_{1}, G_{2}, \cdots$, being those of 4.6 . Then $\psi 1 \leqq 1, \psi f \in \mathcal{F}\left(\mathfrak{T}^{\prime}\right)^{+}, \psi$ is linear and countably additive $\bmod \mathfrak{T}^{\prime}$, and $[\psi f]=\left[\Phi^{*} f\right] \bmod \mathfrak{T}^{\prime}$. Hence $\psi f \in \mathbb{Z}\left(\mathfrak{N}^{\prime}\right)$ if $f \in Z\left(\Re^{\prime}\right)$, and from this an easy induction shows that $\left[\psi^{k} f\right]=\left[\Phi^{*_{k}} f\right] \bmod \mathfrak{N}^{\prime}$ (where $f \in \mathcal{F}\left(\mathfrak{T}^{\prime}\right)^{+}$and $k=0,1,2, \cdots$ ), and that $\psi^{k}$ is countably additive $\bmod \mathfrak{x}^{\prime}$. Further, 
(3)

$$
\left[\sum 2^{-k-1} \psi^{k} \chi X\right]=I(X) \bmod \Re^{\prime}
$$

because (modulo $\left.\Re^{\prime}\right) \quad I(X)=\left[\sum \Phi^{* k} \chi X\right]=U\left[\Phi^{* k} \chi X\right]=U\left[\psi^{k} \chi X\right]$ $=U\left[2^{-k-1} \psi^{k} \chi X\right]=\left[\sum 2^{-k-1} \psi^{k} \chi X\right]$.

Now define, for $X \in \mathfrak{T}^{\prime}$,

$$
m^{*} X=\int_{R} \sum 2^{-k-1} \psi^{k} \chi X d m^{\prime} .
$$

The integrand is $\mathfrak{m}^{\prime}$-measurable, non-negative, and $\leqq 1$, so $m^{*}$ is well defined and is a finite, countably additive measure on $\mathfrak{T}^{\prime}$. We complete this measure as usual, still calling it $m^{*}$, and show

$$
m^{*} Y=0 \text { if and only if } Y \in \Re \text {. }
$$

For if $m^{*} Y=0$, we have $Y \subset X$ where $X \in \Re^{\prime}$ and $m^{*} X=0$. In the definition of $m^{*} X$, the integrand must be zero almost everywhere $\left(m^{\prime}\right)$, which from (3) and (2) gives $I(X) \in \Re^{0}$ and therefore $Y \in \Re$. Conversely, if $Y \in \Re$, $Y \subset X \in \mathfrak{N}^{\prime}$ where $I(X) \in \Re^{0}$, and so $m^{*} X=0$.

The algebra $E$ is now defined exactly as in 5.2 , except that we use the new meaning of $\Re$; and the measure $m^{*}$ on $\mathfrak{T}^{\prime}+\Re$ induces a positive $\sigma$ finite measure $m^{*}$ on $E$. The proof of 5.2(1) no longer applies (as there we used $\Re \subset \oiiint R$ ), but the result itself (the countable chain condition) is a trivial consequence of the existence of $m^{*}$. The operator $\phi$ is defined just as in 5.3; the arguments in $\mathbf{5 . 3}$ and 5.4 apply unchanged, so that $\phi$ is a fully homogeneous cylinder extension of $\phi_{0}$. Finally, by Theorem 2, we replace the measure $m^{*}$ on $E$ by a (positive, $\sigma$-finite) measure $m$ which extends $m_{0}$ on $E_{0}$, and the proof is complete.

\section{REFERENCES}

1. E. Hopf, Ergodentheorie, Berlin, Springer, 1937.

2. D. Maharam, The representation of abstract measure functions, Trans. Amer. Math. Soc. vol. 65 (1949) pp. 279-330.

3. - Decompositions of measure algebras and spaces, Trans. Amer. Math. Soc. vol. 69 (1950) pp. 142-160.

4. - The representation of abstract integrals, Trans. Amer. Math. Soc. vol. 75 (1953) pp. 154-184.

5. - On kernel representation of linear operators, Trans. Amer. Math. Soc. vol. 79 (1955) pp. 229-255.

6. M. H. Stone, Boundedness properties in function lattices, Canad. J. Math. vol. 1 (1949) pp. 176-186.

\footnotetext{
UNIVERSiTY OF MANCHESTER, MANCHESTER, ENGLAND
} 